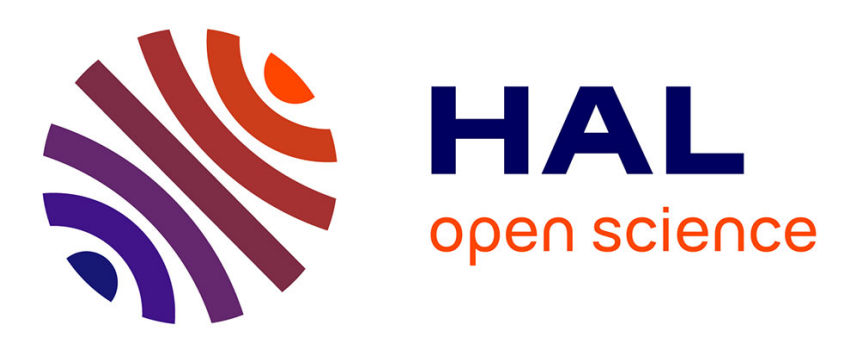

\title{
Structure of ATP citrate lyase and the origin of citrate synthase in the Krebs cycle
}

Koen Verschueren, Clement Blanchet, Jan Felix, Ann A. Dansercoer, Dirk de

Vos, Yehudi Bloch, Jozef van Beeumen, Dmitri Svergun, Irina Gutsche, Savvas Savvides, et al.

\section{To cite this version:}

Koen Verschueren, Clement Blanchet, Jan Felix, Ann A. Dansercoer, Dirk de Vos, et al.. Structure of ATP citrate lyase and the origin of citrate synthase in the Krebs cycle. Nature, 2019, 568 (7753), pp.571-575. 10.1038/s41586-019-1095-5 . hal-02418781

\section{HAL Id: hal-02418781 \\ https://hal.univ-grenoble-alpes.fr/hal-02418781}

Submitted on 24 Nov 2020

HAL is a multi-disciplinary open access archive for the deposit and dissemination of scientific research documents, whether they are published or not. The documents may come from teaching and research institutions in France or abroad, or from public or private research centers.
L'archive ouverte pluridisciplinaire HAL, est destinée au dépôt et à la diffusion de documents scientifiques de niveau recherche, publiés ou non, émanant des établissements d'enseignement et de recherche français ou étrangers, des laboratoires publics ou privés. 


\section{Structure of ATP citrate lyase and the origin of citrate synthase in the Krebs cycle}

Koen H. G. Verschueren ${ }^{1,2}$, Clement Blanchet ${ }^{3}$, Jan Felix ${ }^{4}$, Ann Dansercoer ${ }^{1,2}$, Dirk De Vos ${ }^{5}$, Yehudi Bloch ${ }^{1,2}$, Jozef Van Beeumen ${ }^{6}$, Dmitri Svergun $^{3}$, Irina Gutsche ${ }^{4}$, Savvas N. Savvides ${ }^{1,2} \&$ Kenneth Verstraete $^{1,2 *}$

Across different kingdoms of life, ATP citrate lyase (ACLY, also known as ACL) catalyses the ATP-dependent and coenzyme A (CoA)-dependent conversion of citrate, a metabolic product of the Krebs cycle, to oxaloacetate and the high-energy biosynthetic precursor acetyl-CoA ${ }^{1}$. The latter fuels pivotal biochemical reactions such as the synthesis of fatty acids, cholesterol and acetylcholine ${ }^{2}$, and the acetylation of histones and proteins ${ }^{3,4}$. In autotrophic prokaryotes, ACLY is a hallmark enzyme of the reverse Krebs cycle (also known as the reductive tricarboxylic acid cycle), which fixates two molecules of carbon dioxide in acetyl- $\mathrm{CoA}^{5,6}$. In humans, ACLY links carbohydrate and lipid metabolism and is strongly expressed in liver and adipose tissue $e^{1}$ and in cholinergic neurons ${ }^{2,7}$. The structural basis of the function of ACLY remains unknown. Here we report high-resolution crystal structures of bacterial, archaeal and human ACLY, and use distinct substrate-bound states to link the conformational plasticity of ACLY to its multistep catalytic itinerary. Such detailed insights will provide the framework for targeting human ACLY in cancer $^{8-11}$ and hyperlipidaemia ${ }^{12,13}$. Our structural studies also unmask a fundamental evolutionary relationship that links citrate synthase, the first enzyme of the oxidative Krebs cycle, to an ancestral tetrameric citryl-CoA lyase module that operates in the reverse Krebs cycle. This molecular transition marked a key step in the evolution of metabolism on Earth.

Human ACLY (hACLY) is a 1,101-residue polypeptide forming a functional 0.5-MDa tetramer and featuring an N-terminal citryl-CoA synthetase (CCS) module, consisting of CCS $\beta$ and CCS $\alpha$ regions, and a C-terminal citryl-CoA lyase (CCL) domain ${ }^{14,15}$ (Fig. 1a). To determine the structural basis for the multistep ACLY reaction mechanism $^{16}$ (Extended Data Fig. 1a), we initially performed negative-stain electron microscopy analysis on crystallization-recalcitrant hACLY. These studies revealed that hACLY displays flexible arms around a compact core but also suggested substantial conformational heterogeneity under such experimental conditions (Extended Data Figs. 1b, 9). To facilitate structural studies by X-ray crystallography, we produced a variant of hACLY, termed hACLY-A/B, that lacked the linker region that connects the ancestral ACLY-A and ACLY-B parts (residues 426-486) and which contains the regulatory phosphorylation sites ${ }^{17,18}$ (Fig. 1a, Extended Data Fig. 1c, d). Notably, hACLY-A/B displayed a twofold higher activity in vitro than full-length hACLY (Extended Data Fig. 1e). This indicates that the long linker region between CSS $\beta$ and CSS $\alpha$ might have an auto-inhibitory role, at least in the unphosphorylated state. Subsequent crystal structures to $3.3 \AA$ resolution in space groups $P 1$ and $C 2$ for hACLY-A/B in complex with ADP, citrate and CoA show that the CCL domains of four hACLY chains form an intertwined, D2-symmetric 120-kDa CCL module that serves as the oligomerization platform of the ACLY enzyme (Fig. 1b, Extended Data Fig. 1f, g, Extended Data Table 1). Four CCS $\alpha / \beta$ modules are connected to this CCL module via a long linker that tethers CCS $\alpha$ to a two-helix stalk protruding from the CCL module (Fig. 1a, b, Extended
Data Fig. 1g). The CCL module contains four citrate synthase (CS)like CoA-binding domains ${ }^{19}$. Each of them grasps the adenosine head of CoA with the $3^{\prime}$ phosphoryl group of CoA accommodated at the interface between the CoA-binding domains and a juxtaposed CCS $\alpha / \beta$ module. The phosphopantothenic arms of these CoA molecules bind across the nucleotide-binding motif of the juxtaposed CCS $\alpha$-region and protrude into the CCS active site (Extended Data Fig. 2a, b). At this site, the thiol group of CoA reacts with ATP-derived citryl-phosphate to form citryl-CoA ${ }^{18,20}$. The observed arrangement of hACLY-A/B is further stabilized by few contacts between the CCS module and the CoA-binding domains, and by a $\beta$-hairpin motif in CCS $\alpha$ that contacts the CCL stalk region (Fig. 1b). Superposition of the different hACLY$\mathrm{A} / \mathrm{B}$ crystal structures reveals a range of CCS module conformations with respect to the tetrameric CCL core platform, providing evidence for the extensive structural plasticity of ACLY tetramers around the CCL stalk region (Extended Data Fig. 1h).

We also pursued crystallographic studies of the isolated CCL module of hACLY at high resolution in complex with citrate and CoA, and showed that the CCL protomers can adopt a closed state, in which the CoA-binding domains undergo an approximately $10^{\circ}$ rotation compared to the open state observed in the hACLY-A/B crystal structures (Fig. 1c, Extended Data Fig. 3a-c, Extended Data Table 1, Supplementary Video 1). In the isolated CCL module, CoA molecules adopt a compact conformation in which the phosphopantheine tail folds back across its adenosine head to reach the CCL citrate-binding site at the junction between the CoA-binding domains and the helical bundle core of the CCL module (Extended Data Fig. 3d). The rotation of the CoA-binding domain is necessary to form and shield the CCL active site, as this domain contributes the residues His975, Arg986 and catalytic Asp1026 that bind citrate (Extended Data Fig. 3d, e). These structural analyses establish that after the ATP-driven formation of citryl-CoA at the CCS module, the citryl-thioester head shuttles back by around $35 \AA$ to the citrate-binding site of the CCL module with concomitant closure of the CCL active site to allow cleavage of citryl-CoA (Fig. 1d, Extended Data Fig. 3f, Supplementary Video 2).

Given the diversity of homomeric and heteromeric ACLY assemblies across different kingdoms of life ${ }^{15}$, we sought to determine representative crystal structures for heteromeric ACLY-A/B. Structures of ACLY$\mathrm{A} / \mathrm{B}$ from the methanogenic archaeon Methanosaeta concilii ${ }^{21}$ and the green sulfur bacteria Chlorobium limicola ${ }^{6}$ reveal that prokaryotic ACLY-A/B enzymes are highly similar to human ACLY-A/B and also feature $\operatorname{CCS} \alpha \beta$-hairpins that interact with two-helix stalks extending from the CCL module (Fig. 2, Extended Data Figs. 2c, d, 4, 5, Extended Data Table 1). Moreover, similar to its counterpart in hACLY, the CCL module of C. limicola ACLY can also adopt open and closed states (Extended Data Fig. 3g-j, Extended Data Table 1).

In the ancestral variant of the reverse Krebs cycle found in members of the deep branching bacterial phylum Aquificae, the citrate-cleavage reaction is catalysed by the tandem action of two distinct enzymes

\footnotetext{
${ }^{1}$ Unit for Structural Biology, VIB Center for Inflammation Research, Ghent, Belgium. ${ }^{2}$ Unit for Structural Biology, Department of Biochemistry and Microbiology, Ghent University, Ghent, Belgium. ${ }^{3}$ European Molecular Biology Laboratory (EMBL), Hamburg Outstation c/o DESY, Hamburg, Germany. ${ }^{4}$ University of Grenoble Alpes, CNRS, CEA, CNRS, IBS, Grenoble, France. ${ }^{5}$ Integrated Molecular Plant Physiology Research, Department of Biology, University of Antwerp, Antwerp, Belgium. ${ }^{6}$ Department of Biochemistry and Microbiology, Ghent University, Ghent, Belgium. *e-mail: kenneth.verstraete@ugent.be
} 

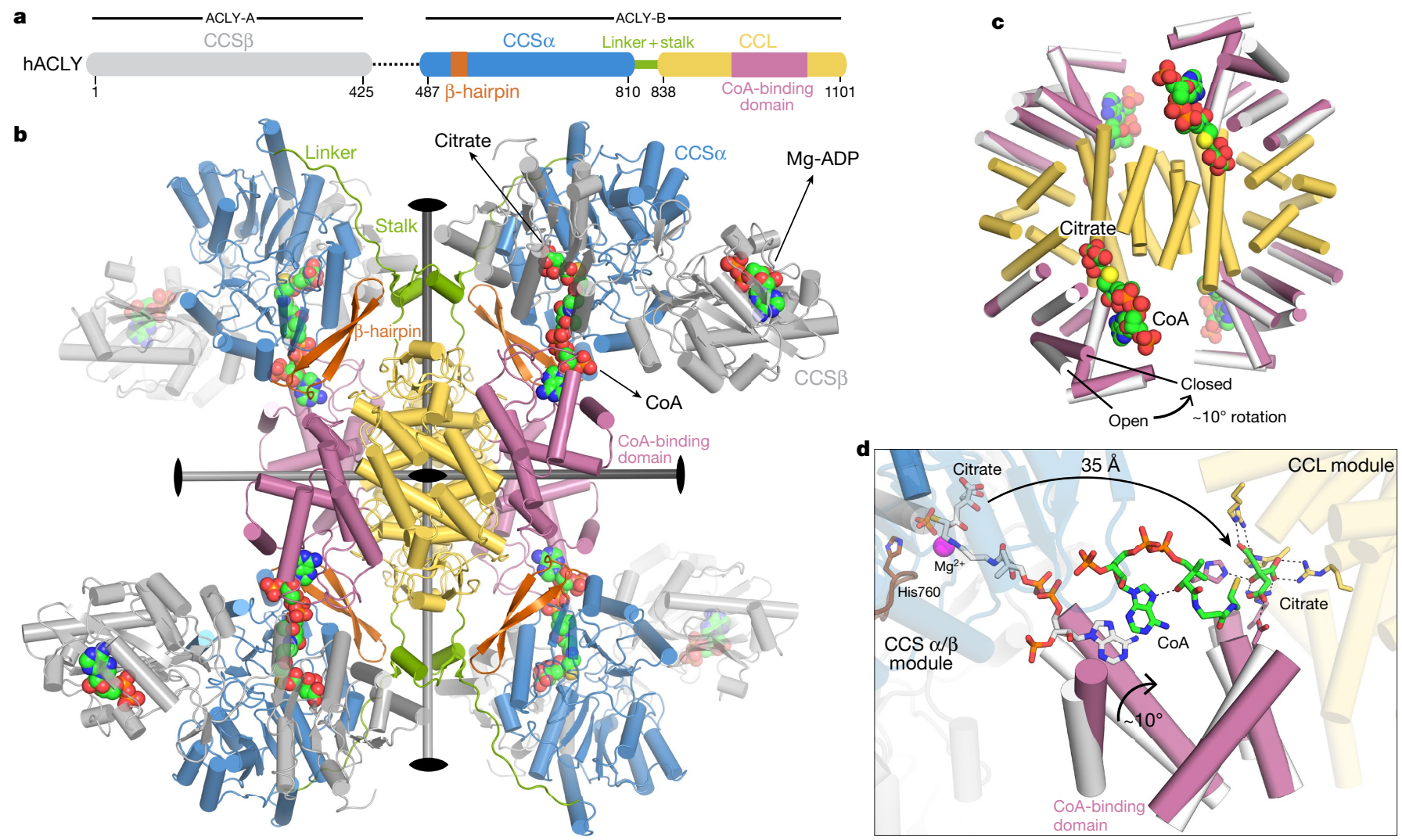

Fig. 1 Structure and mechanism of human ATP citrate lyase. a, Domain organization of hACLY. The dotted line represents the linker between the ancestral ACLY-A and ACLY-B parts. b, Cartoon of a hACLY$\mathrm{A} / \mathrm{B}$ crystal structure in space group $P 1$ coloured according to the scheme in a. The three two-fold axes of the D2 symmetric CCL module are shown.

related to ACLY: $\alpha / \beta$-heteromeric CCS and CCL ${ }^{14,22}$ (Fig. 2). The structure of CCL from Hydrogenobacter thermophilus ${ }^{14}$ shows that stand-alone CCL adopts an intertwined tetrameric structure superimposing with the CCL module of hACLY (Extended Data Fig. 1i, Extended Data Table 1). CCS $\alpha / \beta$ from $H$. thermophilus ${ }^{23}$ is structurally similar to the CCS module of hACLY (root mean square deviation (r.m.s.d.) of $2.3 \AA$ for 330 aligned C $\alpha$ atoms), although in H. thermophilus two CCS $\alpha / \beta$ modules associate to form an $\alpha_{2} \beta_{2}$-heterotetramer as seen for Escherichia coli succinyl-CoA synthetase ${ }^{24}$ (Extended Data Fig. 2e, f, Extended Data Table 1). Thus, the ACLY enzyme arose from the fusion of a tetrameric CCL module with CCS, and by the acquisition of specific structural elements such as the two-helix stalk and interacting $\beta$-hairpins. Corroborating our structural findings, previous binding studies ${ }^{20}$ have shown that hACLY displays four CoA-binding sites. By contrast, C. limicola ACLY can bind to eight CoA molecules: four in the CCL module and four in the CCS modules (Extended Data Fig. 2d). C. limicola ACLY may therefore represent an ancestral ACLY in which the CCS nucleotide-binding motif can still bind the adenosine moiety of CoA, whereas human and archaeal ACLY display bona fide hybrid CoA-binding sites.

Structural superposition of the closed human CCL module with hACLY-A/B showed that the $3^{\prime}$-phosphoryl moiety of CoA would clash with the CCS $\alpha \beta$-hairpin motif (Extended Data Fig. 6a, b). This indicates that the CCS modules need to reorient after transfer of citryl-CoA. Although the structures of human and archaeal ACLY-A/B are equivalent, ACLY-A/B from C. limicola adopts a distinct structural state in which all four CCL active sites are closed, and with the CCS modules reoriented (Fig. 3a, b, Extended Data Figs. 4, 6c). Thus, such
Citrate, CoA and ADP are shown as coloured spheres. c, Superposition of crystal structures for the isolated human CCL module (pink CoA-binding domains) and the CCL module extracted from hACLY-A/B (white CoAbinding domains). d, Structural basis for citryl-CoA shuttling between the CCS and CCL modules.

different functional states illustrate that after rotation of the CoAbinding domains, the CCS module reorients around the CCL module mediated by a pivoting axis defined by the interaction between the CCS $\alpha \beta$-hairpin and the CCL stalk region (Extended Data Fig. 6d-f, Supplementary Videos 3, 4).

To obtain further insights into the structural plasticity of ACLY, we conducted small-angle X-ray scattering (SAXS) experiments on human and C. limicola ACLY-A/B (Extended Data Table 2). Whereas supplementing the SAXS measuring buffer with citrate alone did not result in any notable change in the scattering profile recorded from apoACLY-A/B, the addition of both citrate and CoA induced a transition towards the conformational states observed in the crystal structures (Fig. 3c, d, Extended Data Fig. 6g, h). This shows that ACLY enzymes can oscillate between distinct structural states in a ligand-dependent manner, albeit with differing conformational amplitudes. Subsequent rigid-body modelling of apo-hACLY-A/B suggests an increased structural plasticity around the two-helix stalk region in the absence of CoA (Extended Data Fig. 6g). Additional SAXS data recorded for full-length hACLY indicate a similar structure and ligand-induced rearrangements compared to linker-deleted hACLY-A/B (Extended Data Fig. 6i, j).

CCL - as a stand-alone enzyme or as the core module of ACLYis homologous to CS, the first enzyme in the oxidative Krebs cycle ${ }^{14}$. Prototypical CS is homodimeric and cycles between open and closed states during catalysis ${ }^{19,25}$. Structural comparison of CCL and CS shows that the helical core in tetrameric CCL superimposes well with the helical core in dimeric CS (Fig. 4a, b, Extended Data Fig. 7a). Molecular dissection of CS shows that both the N-terminal and C-terminal halves of the CS protomer exhibit structural and sequence homology to a 

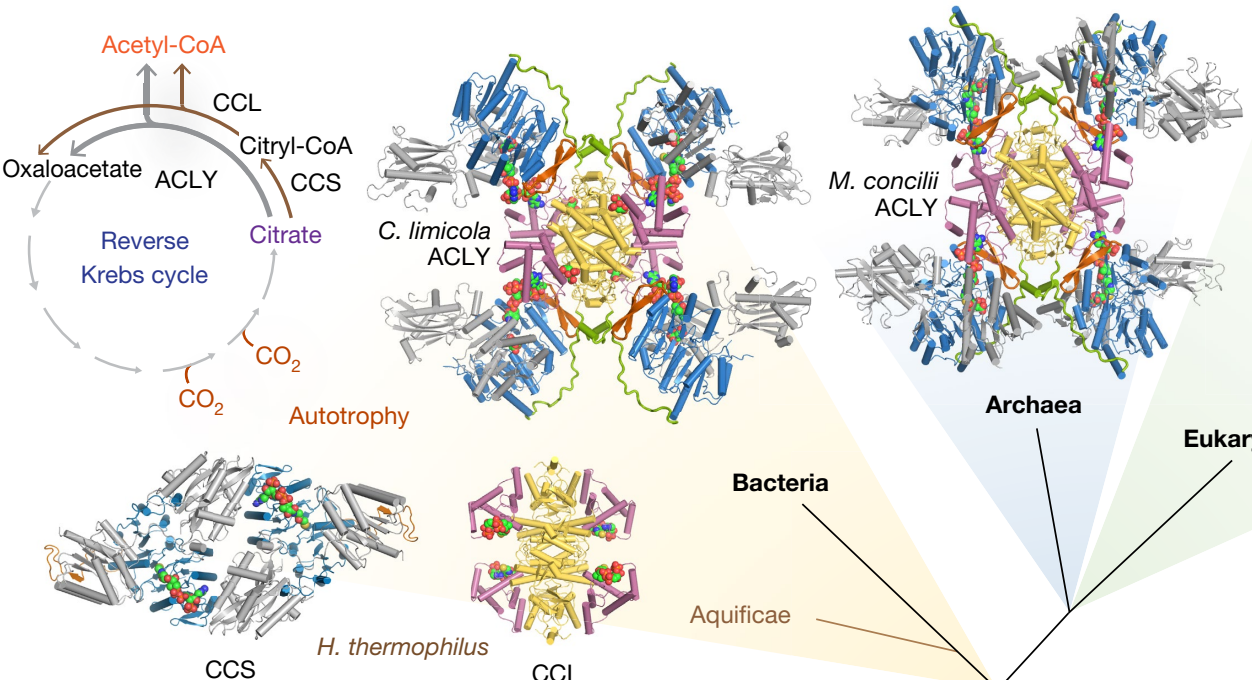

CCS

H. thermophilus

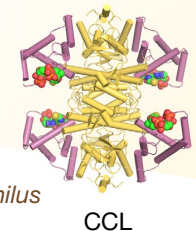

$\mathrm{CCL}$
Fig. 2 Evolutionary origin of ACLY and its distinct metabolic functions. Simplified evolutionary tree illustrating the distribution of ACLY, CCS and CCL enzymes. Determined crystal structures of Homo sapiens, M. concilii and C. limicola ACLY, and H. thermophilus CLL and

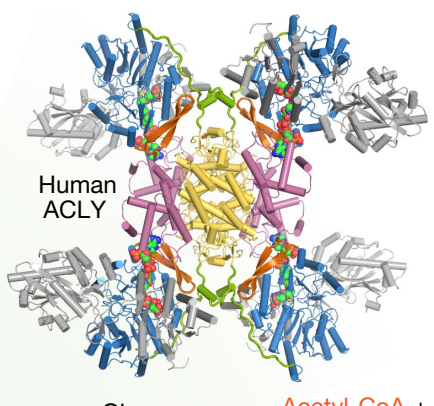

Acetyl-CoA + Archaea
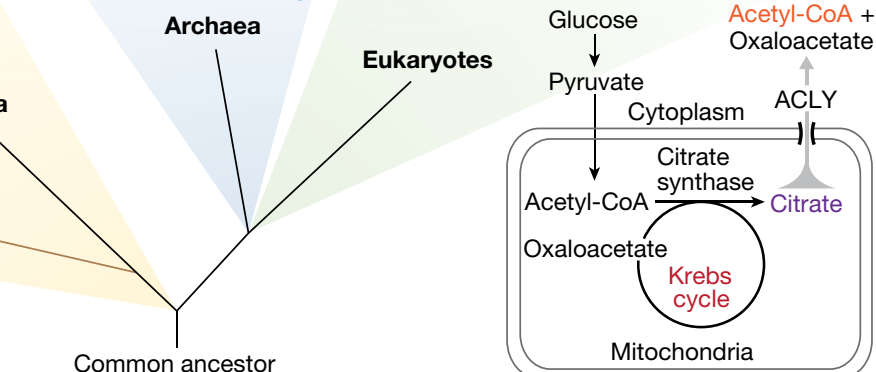

CCL protomer (Extended Data Fig. 7b-g). This provides strong evidence that the CS protomer evolved from the fusion of two ancestral CCL protomers, with the overall structure of the dimeric CS assembly remaining conserved with respect to tetrameric CCL (Fig. 4c). The $\mathrm{N}$-terminal half of CS corresponds to a CCL protomer in which the CoA-binding domain is replaced by a short loop (Extended Data Fig. 7d, g, h). The C-terminal CCL-homology region in CS encompasses the CoA-binding domain, the citrate-binding site and the C-terminal tail that wraps around the adjacent protomer (Extended Data Fig. 7e, g). This fusion event and the introduced internal structural repeat in CS is also apparent from the pseudo-two-fold symmetry axis present in CS, which corresponds to one of the three two-fold symmetry axes in D2-symmetric CCL (Extended Data Fig. 7i, j). Importantly, the molecular transition of CCL to CS indicates that the reverse Krebs cycle, which supports autotrophy, predates the oxidative Krebs cycle

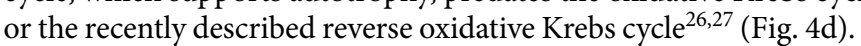

Comparison of high-resolution crystal structures for the substrate-bound CCL module of human and C. limicola ACLY with CS reveals an equivalent active site configuration, indicating a similar aldol

a

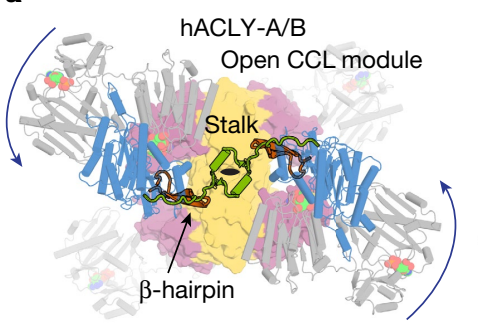

b

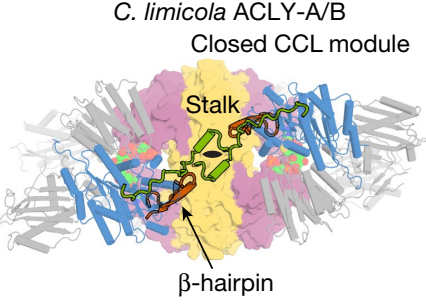

Fig. 3 | ACLY enzymes undergo conformational switching during catalysis. a, b, Top views of human and C. limicola ACLY-A/B illustrating the conformational switching of ACLY. c, d, SAXS profiles for human and C. limicola ACLY-A/B, plotted as the scattered intensity in function of scattering vector $q=4 \pi \sin \theta / \lambda$. (i) Comparison of experimental scattering profiles in HBS buffer (green) and HBS buffer supplemented with citrate (brown); (ii) comparison of experimental scattering profiles in HBS buffer (green) and HBS buffer supplemented with both citrate and CoA (purple); (iii) experimental scattering profiles in HBS buffer overlaid with those reaction mechanism (Fig. 4e-g, Extended Data Fig. 8a-c, Extended Data Table 1). In CS, the catalytic aspartate residue (Asp375 in chicken CS) initiates the enolization of acetyl-CoA by abstracting a C $\alpha$ methyl proton $^{19}$. We propose that during the analogous retro-aldol cleavage of citryl-CoA by hACLY, the corresponding Asp 1026 protonates the acetyl-CoA enolate intermediate, while the main-chain nitrogen of Gly936 is poised to polarize the carbonyl oxygen of the citryl-CoA thioester (Extended Data Fig. 8d). Consistent with its catalytic role, Asp1026 of hACLY engages in a low-barrier hydrogen bond ${ }^{28}$ (distance of $2.4 \AA$ ) with the pro-S carboxylate of the bound citrate molecule (Fig. 4e). Further studies, such as by neutron diffraction, are needed to identify the base abstracting the hydroxyl proton of citryl-CoA to initiate retro-aldol cleavage (Extended Data Fig. 8d). Proposals for the acid responsible for protonating the carbonyl oxygen of oxaloacetate in the reverse reaction by CS have included His $320^{19}$ and $\mathrm{Arg} 329^{29}$, as well as a mechanism that does not involve a direct proton transfer ${ }^{30}$.

Human ACLY is a central component of the citrate shuttle in the liver that transports carbohydrate-derived acetyl-CoA from mitochondria to the cytoplasm for de novo lipogenesis and cholesterol
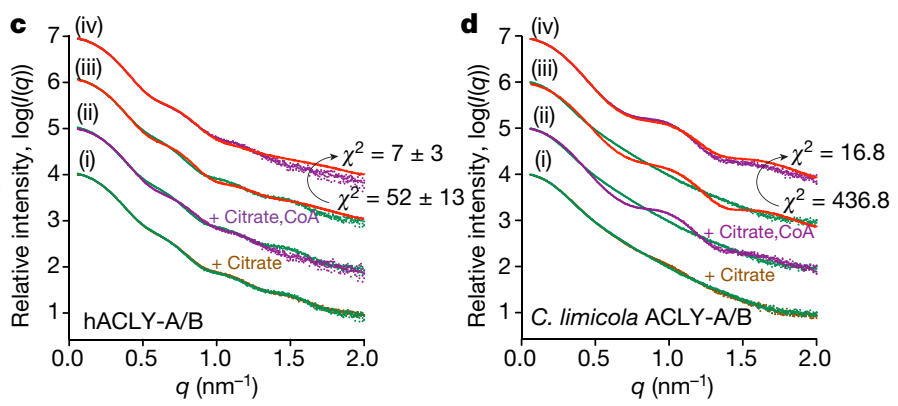

calculated from the substrate-bound crystal structures for human and C. limicola ACLY-A/B (red); and (iv) scattering profiles in HBS buffer supplemented with both citrate and CoA overlaid with scattering profiles calculated from the substrate-bound crystal structures for human and and C. limicola ACLY-A/B (red). Fits were calculated with Crysol 3.0. In $\mathbf{c}$, the reported $\chi^{2}$ values are the mean \pm s.d. for the fits calculated from the different hACLY-A/B crystal structures $(n=4)$ extracted from the $P 1$ and $C 2$ crystal forms, and the best fitting theoretical curve is shown. 

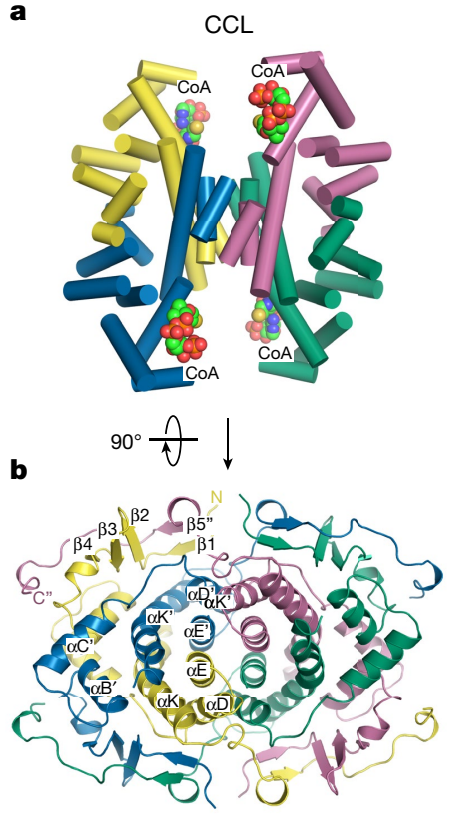

e

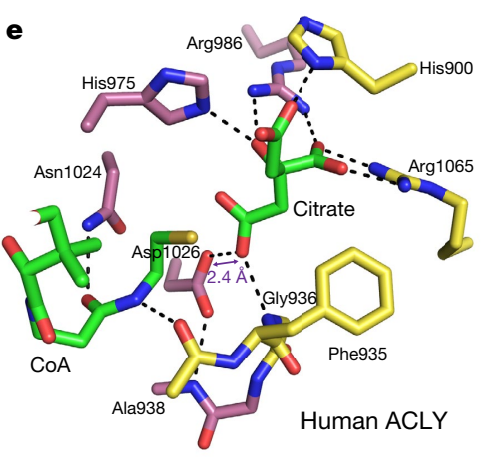

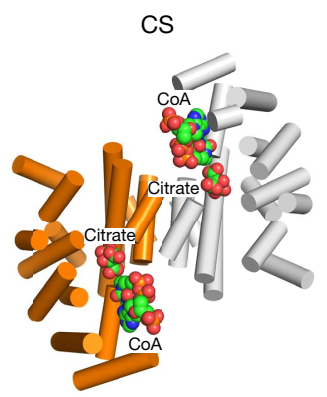

$90^{\circ} \frac{\bigcap}{\uparrow} \downarrow$

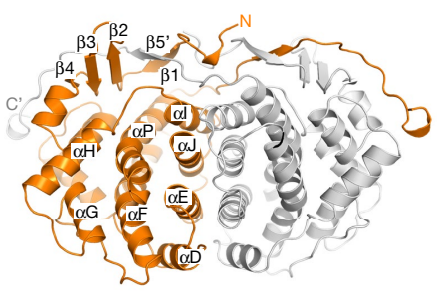

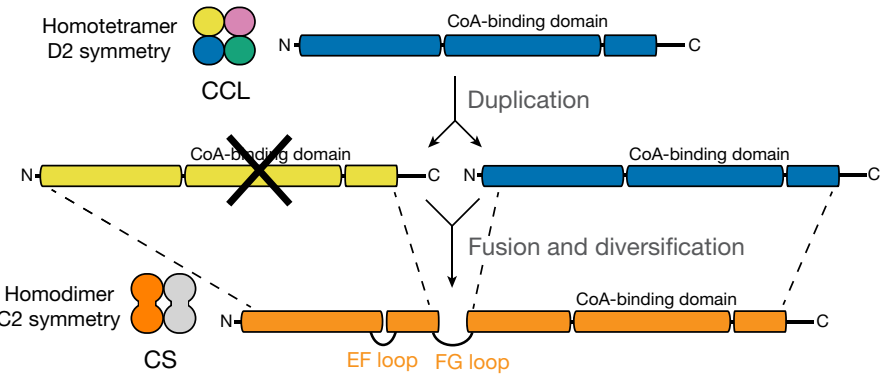

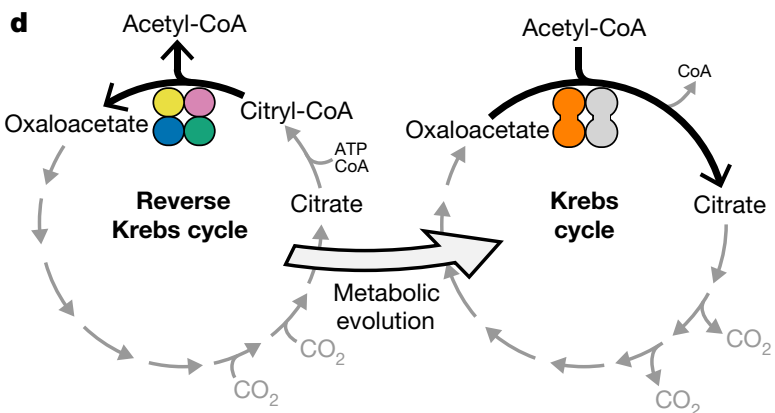

$\mathbf{f}$

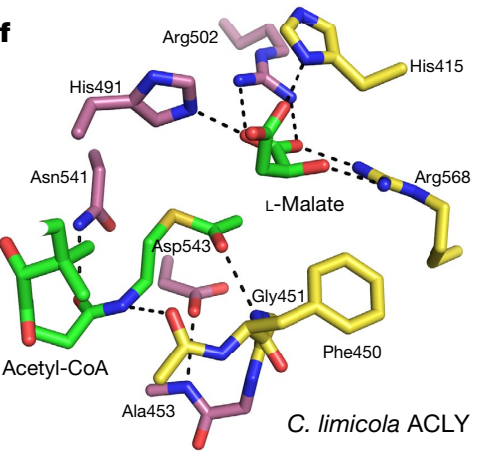

$\mathbf{g}$

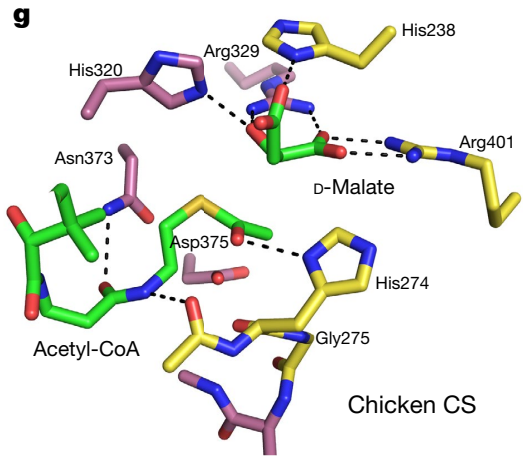

Fig. 4 | Citrate synthase evolved from an ancestral CCL module. a, Cartoon representation of $H$. thermophilus CCL and Pyrococcus furiosus citrate synthase (PDB accession 1AJ8) with CCL and CS coloured by chain. Only helical secondary structure elements are shown. $\mathbf{b}$, View on the helical cores and wrapping of C-terminal tails of CCL and citrate synthase. In this view, the CoA-binding domains are omitted for clarity. c, Proposed evolutionary history of CCL and CS. d, Proposed evolutionary relationship

synthesis. The findings we report here set the stage for unravelling the molecular basis of allosteric regulation of hACLY activity ${ }^{17}$, and for targeting ACLY in cancer ${ }^{8}$ and metabolic diseases ${ }^{12}$. Moreover, the crucial evolutionary relationship between CCL and CS had hitherto remained undetected in sequence-based approaches, and is now poised to reshape our understanding of the evolutionary origins of the oxidative Krebs cycle.

\section{Online content}

Any methods, additional references, Nature Research reporting summaries, source data, statements of data availability and associated accession codes are available at https://doi.org/10.1038/s41586-019-1095-5.

Received: 18 October 2018; Accepted: 8 March 2019; Published online 3 April 2019.

1. Chypre, M., Zaidi, N. \& Smans, K. ATP-citrate lyase: a mini-review. Biochem. Biophys. Res. Commun. 422, 1-4 (2012).

2. Sun, J. et al. BNIP-H recruits the cholinergic machinery to neurite terminals to promote acetylcholine signaling and neuritogenesis. Dev. Cell 34, 555-568 (2015).

3. Wellen, K. E. et al. ATP-citrate lyase links cellular metabolism to histone acetylation. Science 324, 1076-1080 (2009)

4. Sivanand, S. et al. Nuclear acetyl-CoA production by ACLY promotes homologous recombination. Mol. Cell 67, 252-265.e6 (2017). between the reverse Krebs cycle and the oxidative Krebs cycle based on the relationship between CCL and CS. e, Detail of the CCL active site of hACLY in complex with citrate and CoA (PDB 6HXL). The low-barrier hydrogen bond $(2.4 \AA)$ between Asp1026 and bound citrate is indicated. f, Detail of the CCL active site of C. limicola ACLY in complex with L-malate and acetyl-CoA (PDB 6QCL). g, Detail of the active site of chicken CS in complex with D-malate and acetyl-CoA (PDB 4CSC).

5. Hügler, M. \& Sievert, S. M. Beyond the Calvin cycle: autotrophic carbon fixation in the ocean. Ann. Rev. Mar. Sci. 3, 261-289 (2011).

6. Kanao, T., Fukui, T., Atomi, H. \& Imanaka, T. ATP-citrate lyase from the green sulfur bacterium Chlorobium limicola is a heteromeric enzyme composed of two distinct gene products. Eur. J. Biochem. 268, 1670-1678 (2001).

7. Beigneux, A. P. et al. ATP-citrate lyase deficiency in the mouse. J. Biol. Chem. 279, 9557-9564 (2004).

8. Granchi, C. ATP citrate lyase (ACLY) inhibitors: an anti-cancer strategy at the crossroads of glucose and lipid metabolism. Eur. J. Med. Chem. 157, 1276-1291 (2018).

9. Hatzivassiliou, G. et al. ATP citrate lyase inhibition can suppress tumor cell growth. Cancer Cell 8, 311-321 (2005).

10. Migita, T. et al. ATP citrate lyase: activation and therapeutic implications in non-small cell lung cancer. Cancer Res. 68, 8547-8554 (2008).

11. Zaidi, N., Swinnen, J. V. \& Smans, K. ATP-citrate lyase: a key player in cancer metabolism. Cancer Res. 72, 3709-3714 (2012).

12. Pinkosky, S. L., Groot, P. H. E., Lalwani, N. D. \& Steinberg, G. R. Targeting ATP-citrate lyase in hyperlipidemia and metabolic disorders. Trends Mol. Med. 23, 1047-1063 (2017).

13. Pinkosky, S. L. et al. Liver-specific ATP-citrate lyase inhibition by bempedoic acid decreases LDL-C and attenuates atherosclerosis. Nat. Commun. 7, 13457 (2016).

14. Aoshima, M., Ishii, M. \& Igarashi, Y. A novel enzyme, citryl-CoA lyase, catalysing the second step of the citrate cleavage reaction in Hydrogenobacter thermophilus TK-6. Mol. Microbiol. 52, 763-770 (2004).

15. Fatland, B. L. et al. Molecular characterization of a heteromeric ATP-citrate lyase that generates cytosolic acetyl-coenzyme A in Arabidopsis. Plant Physiol. 130, 740-756 (2002) 
16. Fan, F. et al. On the catalytic mechanism of human ATP citrate lyase. Biochemistry 51, 5198-5211 (2012).

17. Potapova, I. A., El-Maghrabi, M. R., Doronin, S. V. \& Benjamin, W. B. Phosphorylation of recombinant human ATP:citrate lyase by cAMP-dependent protein kinase abolishes homotropic allosteric regulation of the enzyme by citrate and increases the enzyme activity. Allosteric activation of ATP:citrate lyase by phosphorylated sugars. Biochemistry 39, 1169-1179 (2000).

18. Hu, J., Komakula, A. \& Fraser, M. E. Binding of hydroxycitrate to human ATP-citrate lyase. Acta Crystallogr. D 73, 660-671 (2017).

19. Remington, S. J. Structure and mechanism of citrate synthase. Curr. Top. Cell. Regul. 33, 209-229 (1992).

20. Sun, T., Hayakawa, K., Bateman, K. S. \& Fraser, M. E. Identification of the citrate-binding site of human ATP-citrate lyase using X-ray crystallography. J. Biol. Chem. 285, 27418-27428 (2010).

21. Barber, R. D. et al. Complete genome sequence of Methanosaeta concilii, a specialist in aceticlastic methanogenesis. J. Bacteriol. 193, 3668-3669 (2011).

22. Hügler, M., Huber, H., Molyneaux, S. J., Vetriani, C. \& Sievert, S. M. Autotrophic $\mathrm{CO}_{2}$ fixation via the reductive tricarboxylic acid cycle in different lineages within the phylum Aquificae: evidence for two ways of citrate cleavage. Environ. Microbiol. 9, 81-92 (2007).

23. Aoshima, M., Ishii, M. \& Igarashi, Y. A novel enzyme, citryl-CoA synthetase, catalysing the first step of the citrate cleavage reaction in Hydrogenobacter thermophilus TK-6. Mol. Microbiol. 52, 751-761 (2004).

24. Bailey, D. L., Fraser, M. E., Bridger, W. A., James, M. N. \& Wolodko, W. T. A dimeric form of Escherichia coli succinyl-CoA synthetase produced by site-directed mutagenesis. J. Mol. Biol. 285, 1655-1666 (1999).

25. Russell, R. J., Ferguson, J. M., Hough, D. W., Danson, M. J. \& Taylor, G. L. The crystal structure of citrate synthase from the hyperthermophilic archaeon pyrococcus furiosus at 1.9 A resolution. Biochemistry 36, $9983-9994$ (1997).

26. Nunoura, T. et al. A primordial and reversible TCA cycle in a facultatively chemolithoautotrophic thermophile. Science 359, 559-563 (2018).

27. Mall, A. et al. Reversibility of citrate synthase allows autotrophic growth of a thermophilic bacterium. Science 359, 563-567 (2018).

28. Usher, K. C., Remington, S. J., Martin, D. P. \& Drueckhammer, D. G. A very short hydrogen bond provides only moderate stabilization of an enzyme-inhibitor complex of citrate synthase. Biochemistry 33, 7753-7759 (1994).

29. van der Kamp, M. W., Perruccio, F. \& Mulholland, A. J. High-level QM/MM modelling predicts an arginine as the acid in the condensation reaction catalysed by citrate synthase. Chem. Commun. (16), 1874-1876 (2008).

30. Aleksandrov, A., Zvereva, E. \& Field, M. The mechanism of citryl-coenzyme A formation catalyzed by citrate synthase. J. Phys. Chem. B 118, 4505-4513 (2014).

Acknowledgements K.V. and Y.B. are post-doctoral research fellows of the Research Foundation - Flanders (FWO) (fellowships 12A5517N and $12 \mathrm{~S} 0519 \mathrm{~N})$. J.F. is supported by an EMBO long-term post-doctoral fellowship
(ALTF441-2017). This work was supported by grants from the FWO to K.V. (1524918N), a Concerted Research Action (GOA) grant from Ghent University to S.N.S. (BOF17-GOA-028), a Hercules Foundation infrastructure grant to S.N.S. (AUGE-11-029), a programme grant from the VIB to S.N.S., and the Horizon 2020 grants: Chap4Resp (to I.G., grant no. 647784), iNext (grant no. 653706) and CALIPSOplus (grant no. 730872). We acknowledge access to experimental facilities and technical support at the following synchrotron radiation facilities: PETRA III (beamlines P12, P13 and P14), SOLEIL (Proxima-2, Swing), ESRF (ID23-1, ID23-2 and ID30-B), SLS (PXI and PXIII). This work used the platforms of the Grenoble Instruct-ERIC Center (ISBG: UMS 3518 CNRS-CEA-UGAEMBL) with support from FRISBI (ANR-10-INSB-05-02) and GRAL (ANR-10 LABX-49-01) within the Grenoble Partnership for Structural Biology (PSB). The electron microscope facility is supported by the Rhône-Alpes Region, the Fondation Recherche Medicale (FRM), Fonds FEDER, the Centre National de la Recherche Scientifique (CNRS), the CEA, the University of Grenoble, EMBL, and the GIS-Infrastrutures en Biologie Sante et Agronomie (IBISA).

Reviewer information Nature thanks Frank M. Raushel and the other anonymous reviewer(s) for their contribution to the peer review of this work.

Author contributions K.H.G.V. conducted crystallographic experiments and refined crystal structures. C.B. analysed SAXS data with contributions from D.S. J.F. and I.G. performed negative-stain electron microscopy analysis of hACLY. A.D. performed molecular cloning and produced recombinant proteins. D.D.V. and J.V.B. initiated studies on hACLY and provided research tools, with contributions from Y.B. S.N.S. contributed to data analysis. K.V. directed and designed the study, conducted crystallographic, SAXS, size-exclusion chromatography coupled to multiangle laser light scattering (SEC-MALLS) and kinetic experiments, refined and analysed crystal structures, analysed SAXS data, and created the figures. K.V. and S.N.S. wrote the manuscript with contributions from all other authors.

Competing interests The authors declare no competing interests.

Additional information

Extended data is available for this paper at https://doi.org/10.1038/s41586019-1095-5.

Supplementary information is available for this paper at https://doi.org/ 10.1038/s41586-019-1095-5.

Reprints and permissions information is available at http://www.nature.com/ reprints.

Correspondence and requests for materials should be addressed to K.V. Publisher's note: Springer Nature remains neutral with regard to jurisdictional claims in published maps and institutional affiliations.

(c) The Author(s), under exclusive licence to Springer Nature Limited 2019 


\section{METHODS}

No statistical methods were used to predetermine sample size. The experiments were not randomized and the investigators were not blinded to allocation during experiments and outcome assessment.

Recombinant protein production and purification. cDNA encoding fulllength human ACLY (hACLY, Uniprot ID P53396-2) was prepared from poly A+ RNA from liver (Clontech) and cloned into the pTrcHis2 vector, in frame with a C-terminal Myc- and His-tag, resulting in pTrcHis2-hACLY (LMBP 11277). To produce a heteromeric form of human ACLY (hACLY-A/B) without the long linker region (residues 426-487), codon-optimized cDNA fragments encoding residues 1-425 (hACLY-A) and residues 488-1101 (hACLY-B) of human ACLY (Uniprot ID P53396-1) were cloned into the pET-Duet vector, resulting in pETDUET-hACLY-A/B (LMBP 11131). The hACLY-B fragment carried a C-terminal His-tag. Codon-optimized cDNA fragments encoding the M. concilii ACLY-A (NCBI ID WP_048131686.1) and ACLY-B (NCBI ID WP_048131683.1) subunits, and the C. limicola ACLY-A (Uniprot ID Q9AQH6) and ACLY-B (Uniprot ID Q9AJC4) subunits were cloned in the pET11a vector interspersed with an E. coli ribosomal-binding sequence ${ }^{6}$, resulting in the bicistronic constructs pET11aMco-ACLY-A/B (LMBP 11132) and pET11a-Cli-ACLY-A/B (LMBP 11125), with the ACLY-B subunits carrying a C-terminal His-tag. Codon-optimized cDNA fragments encoding the $H$. thermophilus CCS $\alpha$ (Uniprot ID Q75VW6) and CCS3 (Uniprot ID D3DK29) subunits were cloned in the pET-DUET vector, resulting in pET-Duet-Hth-CCS $\alpha / \beta$ (LMBP 11134), with the CCS $\beta$ fragment carrying a C-terminal His-tag. A codon-optimized cDNA fragment encoding H. thermophilus CCL (Uniprot ID Q75VX1) was cloned into the pET11a plasmid in frame with a C-terminal His-tag, resulting in pET11a-Hth-CCL (LMBP 11133). Codon-optimized CDNA fragments encoding the CCL core modules of human ACLY (Uniprot ID P53396-1, residues 836-1101) and C. limicola ACLY (Uniprot ID Q9AQH6, residues 351-608) were cloned into the pET15b vector, in frame with a thrombin-cleavable $\mathrm{N}$-terminal His-tag, resulting in expression plasmids pET15b-hCCL (LMBP 11128) and pET15b-Cli-CCL (LMBP 11129), respectively. pTrcHis2-hACLY was expressed in the E. coli $\mathrm{C} 43$ (DE3) strain grown at $28^{\circ} \mathrm{C}$. All other constructs were expressed in the E. coli BL21(DE3) strain. pET11aCli-ACLY-A/B was expressed at $28^{\circ} \mathrm{C}$ and other constructs at $20^{\circ} \mathrm{C}$. Cultures were grown in Luria-Bertani broth and expression was induced by the addition of $1 \mathrm{mM}$ IPTG at an optical density at $600 \mathrm{~nm}$ of $0.6-0.7$. After overnight expression, cultures were collected by centrifugation and bacterial cells were resuspended in IMAC binding buffer, consisting of $50 \mathrm{mM}$ sodium phosphate, $\mathrm{pH} 7.4$ and $150 \mathrm{mM} \mathrm{NaCl}$, supplemented with $1 \mathrm{mM}$ dithiothreitol (DTT). To prevent proteolysis in the long linker region in full-length hACLY, cOmplete Protease Inhibitor Cocktail without EDTA (Roche) was added. Bacterial cells were lysed by sonication and insoluble material was removed by centrifugation. The resulting supernatant was clarified using a $0.22-\mu \mathrm{m}$ filter and loaded onto a cOmplete His-tag (Roche) or Ni Sepharose column equilibrated with IMAC binding buffer. The IMAC column was washed and recombinant His-tagged proteins were eluted with binding buffer supplemented with increasing concentrations of imidazole. Elution fractions containing the His-tagged protein of interest were pooled and concentrated using ultracentrifugation. Proteins were further purified by size-exclusion chromatography (SEC) using HiLoad 16/600 Superdex 200 and Superose 6 (Increase) columns. As a SEC running buffer, $20 \mathrm{mM}$ HEPES, pH 7.4, $150 \mathrm{mM} \mathrm{NaCl}$ supplemented with $1 \mathrm{mM}$ DTT was used; for the purification of ACLY enzymes, $20 \mathrm{mM}$ citrate, pH 6.0, $150 \mathrm{mM} \mathrm{NaCl}$ supplemented with $1 \mathrm{mM}$ DTT was used. ACLY enzymes were purified at $4{ }^{\circ} \mathrm{C}$. The $\mathrm{N}$-terminal His-tag of the purified CCL core modules of human ACLY and C. limicola ACLY was removed using thrombin, and following overnight incubation, the digestion mixture was injected on a SEC column. Elution fractions corresponding to the protein of interest were either used immediately or stored at $-80^{\circ} \mathrm{C}$ until further use.

Enzymatic activity assays. Initial reaction rates for hACLY-A/B, hACLY and the hACLY(His760Ala) mutant in relation to ATP concentration were measured using the malate dehydrogenase assay ${ }^{31}$. Assays were set up in transparent Nunc 96-well plates using a total reaction volume of $250 \mu$ l. The reaction buffer contained $20 \mathrm{mM}$ Tris buffer, $\mathrm{pH} 8.5,20 \mathrm{mM}$ citrate, $10 \mathrm{mM} \mathrm{MgCl} 2,0.5 \mathrm{mM} \mathrm{CoA}, 4 \mathrm{mM}$ DTT, $0.2 \mathrm{mM} \mathrm{NADH}, 4 \mathrm{U} \mathrm{ml}^{-1}$ malate dehydrogenase (Roche) and $1.0 \mu \mathrm{g} \mathrm{ml}^{-1}$ hACLY-A/B or $1.6 \mu \mathrm{g} \mathrm{ml}^{-1} \mathrm{hACLY}$. The reaction was started by the addition of ATP. Oxidation of NADH was followed by measuring the absorbance at $340 \mathrm{~nm}$ in function of time at $37^{\circ} \mathrm{C}$ using a FLUOstar Omega microplate reader (BMG Labtech). Protein concentrations were determined using the absorbance at $280 \mathrm{~nm}$ and theoretical extinction coefficients. For NADH, a molar extinction coefficient of $6,220 \mathrm{M}^{-1} \mathrm{~cm}^{-1}$ was used. Kinetic data were analysed in GraphPad Prism and initial reaction rates were fitted to a Michaelis-Menten equation to obtain the parameters $K_{\mathrm{m}}$ and $k_{\text {cat. }}$. Kinetic data for hACLY-A/B and hACLY were measured from four different protein batches.

Crystallization and structure determination. Purified protein samples were concentrated to $5-15 \mathrm{mg} \mathrm{ml}^{-1}$. For co-crystallization experiments with ligands, the protein samples were supplemented with $50 \mathrm{mM}$ citrate and/or $10 \mathrm{mM} \mathrm{CoA}$ and $50 \mathrm{mM} \mathrm{Mg}$-ADP. Nanolitre-scale vapour diffusion crystallization experiments were set up at $293 \mathrm{~K}$ or $277 \mathrm{~K}$ using commercially available sparse matrix crystals screens (Molecular Dimensions, Hampton Research) and a Mosquito crystallization robot (TTP Labtech). Promising hits were further optimized using gradient optimization in 96-well and 24-well crystallization plates. These optimized conditions are listed in Extended Data Table 1. Crystals were cryoprotected using a quick soak in mother liquid supplemented with the respective cryosolution (Extended Data Table 1) and ligands when appropriate. Crystals were cryo-cooled by plunging into liquid nitrogen. X-ray diffraction measurements were conducted at synchrotron radiation facilities PETRA III (beamlines P13, P14), SOLEIL (Proxima 2A), ESRF (ID23-1, ID23-2, ID30-B) and SLS (PXI, PXIII). All data were integrated and scaled using the XDS suite ${ }^{32}$ and AIMLESS ${ }^{33}$ and data quality was analysed by Phenix. xtriage $^{34}$. To solve the initial structure of ACLY-A/B from C. limicola, molecular replacement was performed with $\mathrm{Phaser}^{35}$ using the structure of $\mathrm{N}$-terminal truncated human ACLY as a search model ${ }^{20}$. After placement of four search CCS modules, and initial refinement the CCL core module was built manually in the remaining difference density using the homology with CS as a structural guide. Parrot ${ }^{36}$ density modification and NCS averaging was used to improve the quality of initial electron density maps. Subsequent structures for human and $M$. concilii ACLY-A/B, human and C. limicola CCL modules, and H. thermophilus CCL, were solved by molecular replacement using derived search models. For $H$. thermophilus $\mathrm{CCS} \alpha / \beta$, search models for the CCS $\alpha$ and $\operatorname{CCS} \beta$ subunits were prepared from homologous succinyl-CoA synthetase structures (PDB 2YV2 for the CCS $\alpha$ subunit and PDB 3UFX for the CCS $\beta$ subunit). Model (re)building was performed in $\mathrm{Coot}^{37}$ and refinement of coordinates and atomic displacement parameters was performed in PHENIX ${ }^{38}$ and autoBUSTER (https://www.globalphasing.com/ buster/). Model and map validation tools in Coot and the PHENIX suite, the CCP4 package ${ }^{39}$ and the PDB_REDO server ${ }^{40}$ were used throughout the work flow to guide improvement and to validate the quality of crystallographic models.

Small-angle $X$-ray scattering data collection and analysis. In-solution SAXS data for C. limicola ACLY-A/B, linker-deleted hACLY-A/B and full-length hACLY were measured on the P12 beamline of EMBL at the Petra III storage ring (DESY, Hamburg). Protein sample ( $50 \mu \mathrm{l}$ of approximately $\left.20 \mathrm{mg} \mathrm{ml}^{-1}\right)$ in HBS $(20 \mathrm{mM}$ HEPES, $150 \mathrm{mM} \mathrm{NaCl}$, pH 7.2) was injected onto an Agilent $4.6 \times 300 \mathrm{~mm}$ Bio SEC- 3 column with $300 \AA$ pore size, and with HBS as running buffer at a flow speed of $0.4 \mathrm{ml} \mathrm{min}^{-1}$ at $20^{\circ} \mathrm{C}$. The scattering data were collected in continuous flow mode with $1 \mathrm{~s}$ exposure time per frame. To collect SAXS data in the presence of citrate, or both citrate and CoA, the SEC-SAXS buffer was supplemented with $50 \mathrm{mM}$ citrate, $\mathrm{pH} 7.2$, or both $50 \mathrm{mM}$ citrate, $\mathrm{pH}$ 7.2, and $2 \mathrm{mM} \mathrm{CoA}$. Before injection, ACLY samples were incubated with citrate alone, or both citrate and CoA. The program CHROMIXS ${ }^{41}$ was used to select the buffer and sample frames from the collected SEC-SAXS data. Overall parameters were calculated using the ATSAS suite version $2.8^{42}$. Calculation of theoretical scattering curves and fitting to experimental scattering data was performed with Crysol, Crysol 3.0 and FoXS. Rigid-body modelling of in-solution scattering data for linker-deleted hACLY-A/B was performed using MultiFoXS ${ }^{43}$ using a crystal structure for hACLY-A/B in space group $C 2$ as a starting model. The presented two-state SAXS model for apohACLY-A/B in HBS $\left(\chi^{2}=2.8\right.$, and with $w_{1}=0.58$ and $\left.w_{2}=0.42\right)$ was obtained by defining the CCS modules and central CCL module as rigid bodies, with residues 808 to 810 and residues 821 to 824 in each CCS-CCL linker region defined as flexible. The presented single-state SAXS model for hACLY-A/B in HBS supplemented with both citrate and $\operatorname{CoA}\left(\chi^{2}=2.8\right)$ was obtained by defining the CCS modules and central CCL module as rigid bodies with residues 808-811 in each CCS-CCL linker region defined as flexible. This SAXS-model for hACLY-A/B was then used to model full-length hACLY in HBS supplemented with both citrate and CoA using AllosMOD-FoXS ${ }^{44}$, resulting in a $\chi^{2}$ value of 5.5. Initial SAXS data on C. limicola ACLY-A/B in HBS buffer without substrates were measured in SEC-SAXS mode on the SWING beamline at the SOLEIL Synchrotron (Gif-sur-Yvette, France) and analysed in Foxtrot (developed at Synchrotron SOLEIL and provided by Xenocs, Sassenage, France).

Negative-stain electron microscopy. Purified full-length hACLY $(3 \mu \mathrm{l})$ supplemented with $2 \mathrm{mM} \mathrm{CoA}$ was applied to the clean side of carbon on a carbon-mica interface and stained with $2 \%(\mathrm{w} / \mathrm{v})$ sodium silicotungstate (SST). Images were recorded on a FEI Tecnai T12 microscope operated at $120 \mathrm{kV}$ with a Gatan Orius 1000 camera, at a nominal magnification of $29,000 \times$, corresponding to a pixel size of $2.0 \AA$ at the object scale. Semi-automatic particle selection on a few micrographs using BOXER ${ }^{45}$ with a box size of 180 pixels resulted in an initial data set containing 9881 particles. Subsequent image analysis was performed in RELION2.1 ${ }^{46}$. After CTF estimation followed by $2 \mathrm{D}$ reference-free classification, a set of classes representing different views of hACLY was used as input for automated particle picking, resulting in a data set of 25,832 particles. Following $2 \mathrm{D}$ reference-free classification, particles from the best 6 classes were used to make an initial model with applied C2 symmetry. A subsequent 3D refinement was performed by using 
the initial model as an input. The resulting refined model was used to re-pick particles employing the fast projection matching (FPM) $\operatorname{method}^{47,48}$, resulting in an extended dataset of 44,817 particles that was further processed in RELION2.1. After two rounds of 2D reference-free classification and cleaning, a final dataset of 27,293 particles was subjected to 3D classification using C2 symmetry. Out of the four resulting classes, two showed highly similar features and together accounted for $57.1 \%$ of all particles $(15,617)$. A final 3D refinement was performed on the particles within these two 3D classes, using an averaged 3D model generated from their corresponding maps in Chimera ${ }^{49}$. This resulted in a final 3D map with a resolution of $26.6 \AA$ according to the Fourier shell correlation (FSC) $=0.143$ criterion. Multi-angle laser light scattering. hACLY-A/B $(100 \mu \mathrm{l})$ was injected onto a Superdex 200 Increase 10/300 GL column (GE Healthcare), with $20 \mathrm{mM}$ citrate, $\mathrm{pH} 7.4$, and $150 \mathrm{mM} \mathrm{NaCl}$ as running buffer at $0.5 \mathrm{ml} \mathrm{min}^{-1}$, coupled to an online UV-detector (Shimadzu), a multi-angle light scattering miniDAWN TREOS instrument (Wyatt) and a Optilab T-rEX refractometer (Wyatt) at $25^{\circ} \mathrm{C}$. A refractive index increment $(d n / d c)$ value of $0.185 \mathrm{ml} \mathrm{g}^{-1}$ was used for protein concentration and molecular mass determination. Data were analysed using the ASTRA6 software (Wyatt). Correction for band broadening was applied using parameters derived from BSA ( $2 \mathrm{mg} \mathrm{ml}^{-1}$, Pierce) injected under identical running conditions. Structure and sequence analysis. Structures were superimposed with Chimera ${ }^{49}$. Sequence alignments were created using Clustal Omega ${ }^{50}$ and formatted with ESPript $^{51}$. Secondary structures elements of crystallographic structures were assigned with $\mathrm{DSSP}^{52,53}$. Figures containing structural models were prepared in PyMOL (version 2.0; https://pymol.org/2/).

Reporting summary. Further information on research design is available in the Nature Research Reporting Summary linked to this paper.

\section{Data availability}

Protein expression constructs generated in this study are available via the BCCM/ GeneCorner Plasmid Collection (http://bccm.belspo.be) through the following accession codes: LMBP 11277 (pTrcHis2-hACLY), LMBP 11131 (pET-DUEThACLY-A/B), LMBP 11132 (pET11a-Mco-ACLY-A/B), LMBP 11133 (pET11a-HthCCL), LMBP 11134 (pET-Duet-Hth-CCS $\alpha / \beta$ ), LMBP 11125 (pET11a-Cli-ACLYA/B), LMBP 11128 (pET15b-hCCL) and LMBP 11129 (pET15b-Cli-CCL). X-ray crystallographic coordinates and structure factors have been deposited in the Protein Data Bank (PDB) with accession codes 6HXH (hACLY-A/B in space group P1), 6QFB (hACLY-A/B in space group C2), 6HXI (M. concilii ACLY-A/B), 6HXJ (C. limicola ACLY-A/B), 6HXK (CCL module of hACLY, space group $P 2_{1} 2_{1} 2_{1}$ ), 6HXL (CCL module of hACLY, space group $P 2_{1}$ ), $6 \mathrm{HXM}$ (CCL module of hACLY, space group $C 222_{1}$ ), $6 \mathrm{HXN}$ (CCL module of $C$. limicola ACLY, space group $P 3_{1} 21$ ), 6HXO (CCL module of C. limicola ACLY, space group $P 2_{1}$ ), 6QCL (CCL module of C. limicola ACLY in complex with acetyl-CoA and L-malate), $6 \mathrm{HXP}(H$. thermophilus CCL) and 6HXQ (H. thermophilus CCS). SAXS data and models have been deposited in the Small Angle Scattering Biological Data Bank with accession codes SASDE36, SASDE46 and SASDE56 for hACLY-A/B; SASDFA3, SASDFB3 and SASDFC3 for hACLY; and SASDE66, SASDE76 and SASDE86 for C. limocola ACLY-A/B. Source Data for the SEC-MALLS analysis of hACLY-A/B (Extended Data Fig. 1d) and for the enzymatic assays for hACLY and hACLY-A/B (Extended
Data Fig. 1e) are available online. Data are available from the corresponding author(s) upon reasonable request.

31. Linn, T. C. \& Srere, P. A. Identification of ATP citrate lyase as a phosphoprotein. J. Biol. Chem. 254, 1691-1698 (1979).

32. Kabsch, W. XDS. Acta Crystallogr. D 66, 125-132 (2010).

33. Evans, P. R. An introduction to data reduction: space-group determination, scaling and intensity statistics. Acta Crystallogr. D 67, 282-292 (2011).

34. Zwart, P., Grosse-Kunstleve, R. \& Adams, P. Xtriage and Fest: automatic assessment of $\mathrm{X}$-ray data and substructure structure factor estimation. CCP4 Newsl. 43, 27-35 (2005).

35. McCoy, A. J. et al. Phaser crystallographic software. J. Appl. Crystallgr. 40, 658-674 (2007).

36. Cowtan, K. Recent developments in classical density modification. Acta Crystallogr. D 66, 470-478 (2010).

37. Emsley, P., Lohkamp, B., Scott, W. G. \& Cowtan, K. Features and development of Coot. Acta Crystallogr. D 66, 486-501 (2010).

38. Afonine, P. V. et al. Towards automated crystallographic structure refinement with phenix.refine. Acta Crystallogr. D 68, 352-367 (2012).

39. Winn, M. D. et al. Overview of the CCP4 suite and current developments. Acta Crystallogr. D 67, 235-242 (2011).

40. Joosten, R. P., Long, F., Murshudov, G. N. \& Perrakis, A. The PDB REDO server for macromolecular structure model optimization. IUCrJ 1, 213-220 (2014).

41. Panjkovich, A. \& Svergun, D. I. CHROMIXS: automatic and interactive analysis of chromatography-coupled small-angle X-ray scattering data. Bioinformatics $\mathbf{3 4}$, 1944-1946 (2018).

42. Franke, D. et al. ATSAS 2.8: a comprehensive data analysis suite for small-angle scattering from macromolecular solutions. J. Appl. Crystallogr. 50, 1212-1225 (2017).

43. Schneidman-Duhovny, D., Hammel, M., Tainer, J. A. \& Sali, A. FoXS, FoXSDock and MultiFoXS: single-state and multi-state structural modeling of proteins and their complexes based on SAXS profiles. Nucleic Acids Res. $\mathbf{4 4}$ (W1), W424-W429 (2016)

44. Guttman, M., Weinkam, P., Sali, A. \& Lee, K. K. All-atom ensemble modeling to analyze small-angle X-ray scattering of glycosylated proteins. Structure $\mathbf{2 1}$, 321-331 (2013).

45. Ludtke, S. J., Baldwin, P. R. \& Chiu, W. EMAN: semiautomated software for high-resolution single-particle reconstructions. J. Struct. Biol. 128, 82-97 (1999).

46. Scheres, S. H. RELION: implementation of a Bayesian approach to cryo-EM structure determination. J. Struct. Biol. 180, 519-530 (2012).

47. Estrozi, L. F. \& Navaza, J. Fast projection matching for cryo-electron microscopy image reconstruction. J. Struct. Biol. 162, 324-334 (2008).

48. Estrozi, L. F. \& Navaza, J. Ab initio high-resolution single-particle 3D reconstructions: the symmetry adapted functions way. J. Struct. Biol. 172, 253-260 (2010).

49. Pettersen, E. F. et al. UCSF Chimera-a visualization system for exploratory research and analysis. J. Comput. Chem. 25, 1605-1612 (2004).

50. Sievers, F. et al. Fast, scalable generation of high-quality protein multiple sequence alignments using Clustal Omega. Mol. Syst. Biol. 7, 539 (2011).

51. Gouet, P., Robert, X. \& Courcelle, E. ESPript/ENDscript: extracting and rendering sequence and $3 \mathrm{D}$ information from atomic structures of proteins. Nucleic Acids Res. 31, 3320-3323 (2003).

52. Kabsch, W. \& Sander, C. Dictionary of protein secondary structure: pattern recognition of hydrogen-bonded and geometrical features. Biopolymers $\mathbf{2 2}$, 2577-2637 (1983).

53. Touw, W. G. et al. A series of PDB-related databanks for everyday needs. Nucleic Acids Res. 43, D364-D368 (2015). 

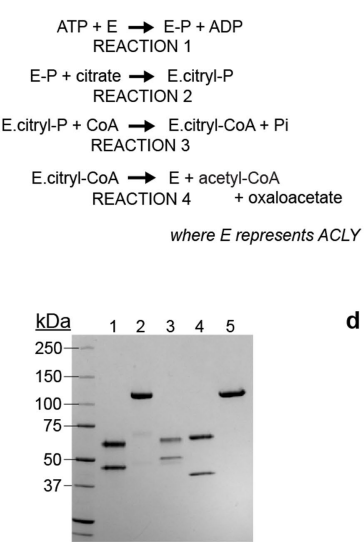

d

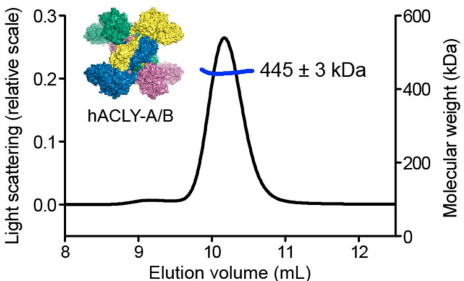

b

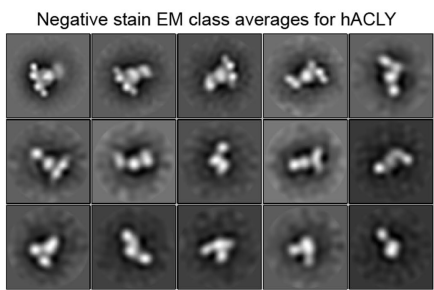

RGLION2.1 Initial 3D model
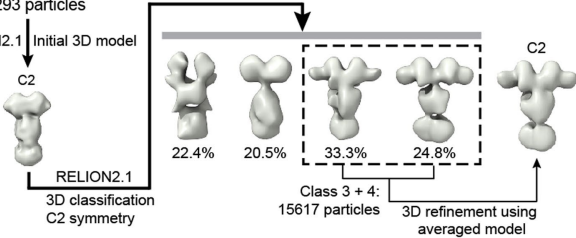

e

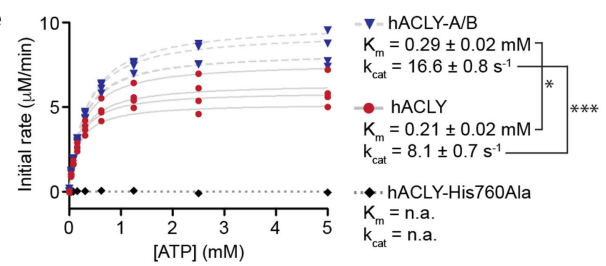

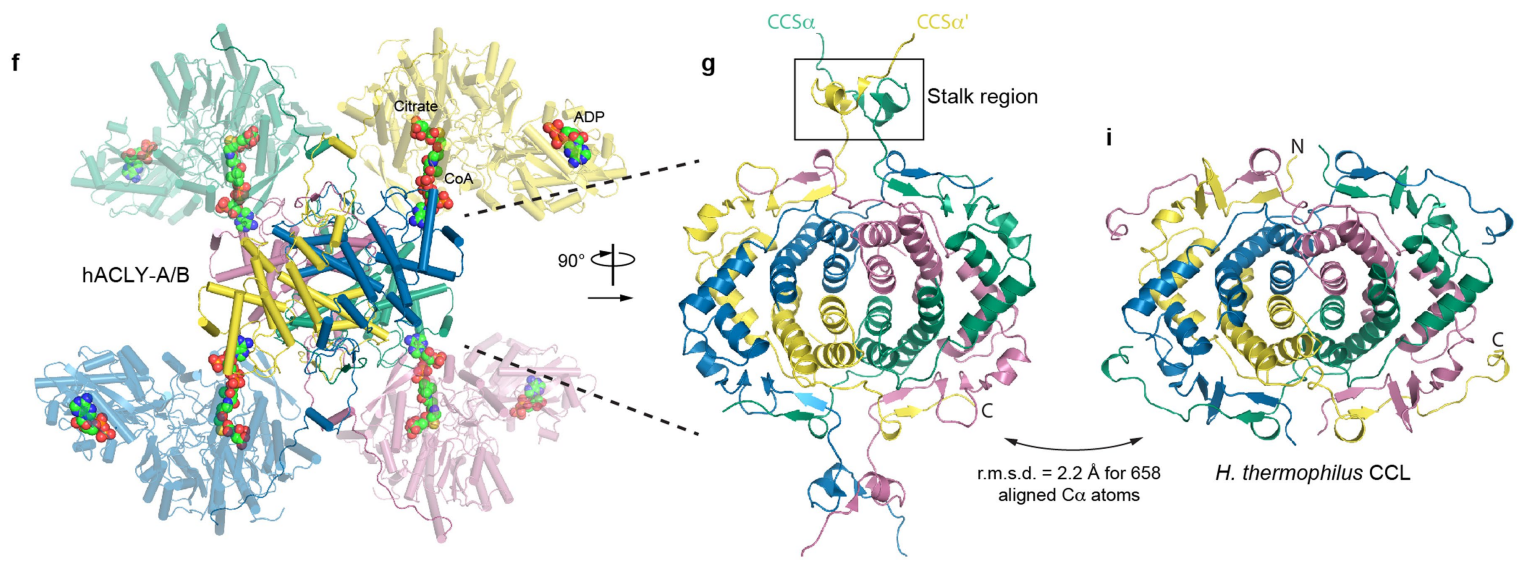

h
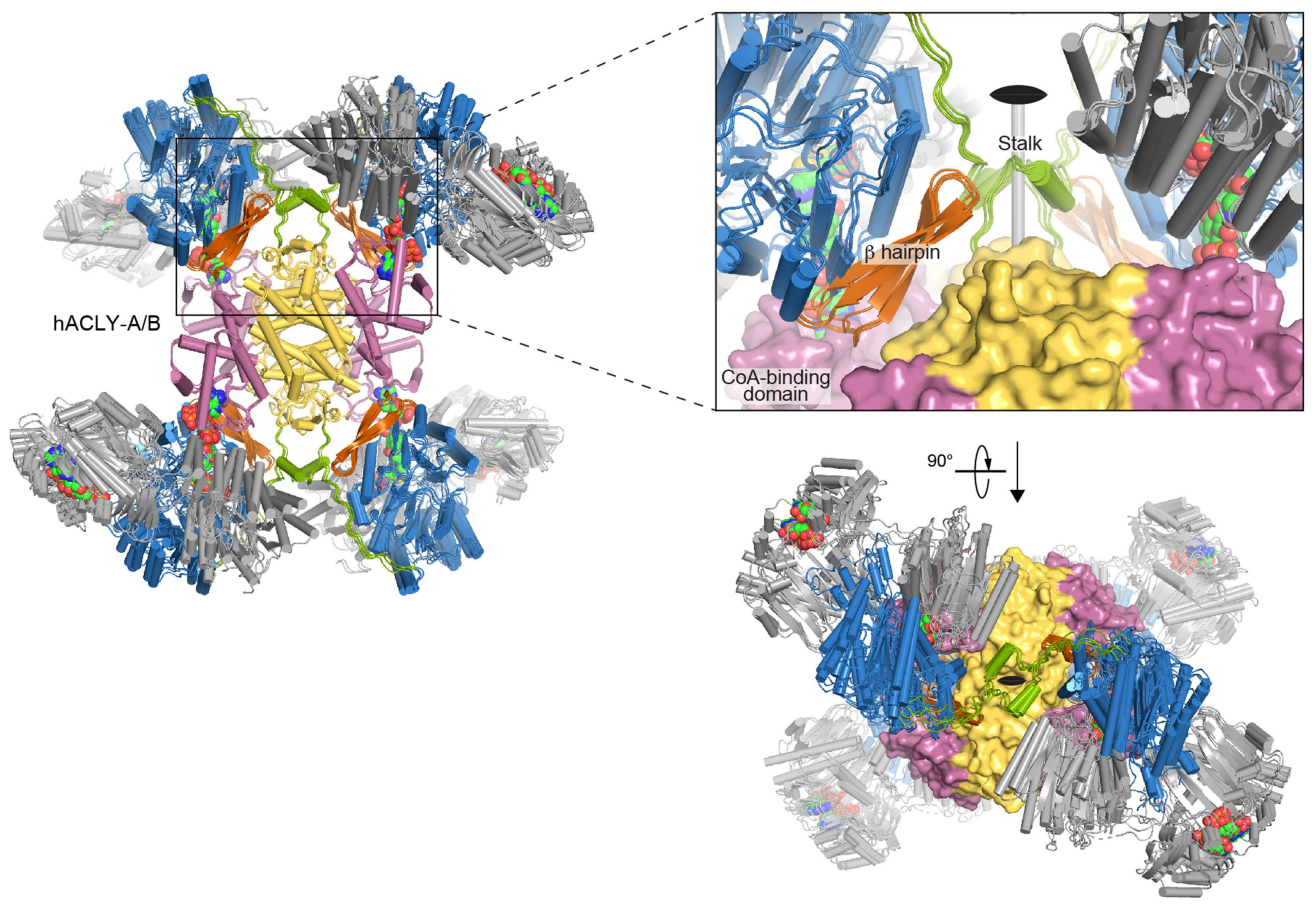

Extended Data Fig. 1 | See next page for caption. 
Extended Data Fig. 1 | Structure of the human ACLY. a, Reaction scheme for ACLY. In the first step, ACLY undergoes autophosphorylation at His760. Citryl-phosphate (citryl-P) and citryl-CoA form non-covalent enzyme-intermediate complexes. $\mathbf{b}$, Left, representative class averages for hACLY as obtained by negative-stain electron microscopy. The size of the box is $40 \times 40 \mathrm{~nm}$. Right, flowchart of the $3 \mathrm{D}$ reconstruction in C2 symmetry. Negative-stain electron microscopy analysis was performed on a single sample of purified hACLY $(n=1)$. SDG, stochastic gradient descent. c, Coomassie-stained SDS-PAGE gel for recombinantly produced ACLY enzymes. Lane 1, hACLY-A/B; lane 2, hACLY; lane 3, M. concilii ACLY-A/B; lane 4, C. limicola ACLY-A/B; lane 5, hACLY(His760Ala). In this study, each protein was purified several times, and the electrophoretic profile of each sample in the gel shown is representative for different protein batches. For gel source data, see Supplementary Fig. 1. d, Sizeexclusion chromatography (SEC) elution profile of hACLY-A/B plotted as the light scattering intensity at $90^{\circ}$ in function of the elution volume. The reported molecular mass by multiangle laser light scattering (MALLS) represents the average molecular mass \pm s.d. across the elution peak. The theoretical mass for hACLY-A/B is $462 \mathrm{kDa}$. Number of samples for hACLY-A/B analysed: $n=1$. e, Reaction rates for hACLY-A/B, hACLY and hACLY(His760Ala) plotted as a function of ATP concentration. For hACLY-A/B and hACLY, data replicates ( $n=4$, in which $n$ represents a different protein batch) were fitted by a Michaelis-Menten equation and the obtained Michaelis constant $\left(K_{\mathrm{m}}\right)$ and turnover number $\left(k_{\mathrm{cat}}\right)$ values (mean + s.e.m) are shown. The kinetic parameters for hACLY-A/B and hACLY are significantly different via two-tailed unpaired $t$-tests: $P=0.0002$ (comparing $k_{\text {cat }}$ ); and $P=0.0156$ (comparing $K_{\mathrm{m}}$ ). For the hACLY(His760Ala) mutant, the number of replicate batches: $n=1$. f, Representative crystal structure for hACLY-A/B extracted from the $P 1$ crystal form and coloured by chain. Bound substrates are shown as coloured spheres. g, View on the helical bundle core of the CCL module with the protruding two-helix stalk regions indicated. CoA-binding domains are omitted for clarity. $\mathbf{h}$, Overlay of the four hACLY-A/B crystal structures extracted from the $P 1$ and $C 2$ crystal forms. The overlay is based on the superposition of the CCL modules. Structures are coloured according to the scheme in Fig. 1a. A zoom-in view shows the structural plasticity around the two-helix stalk region. i, View on the helical bundle core of CCL from $H$. thermophilus coloured by chain. The $\mathrm{N}$ and $\mathrm{C}$ termini of a single chain are indicated. 


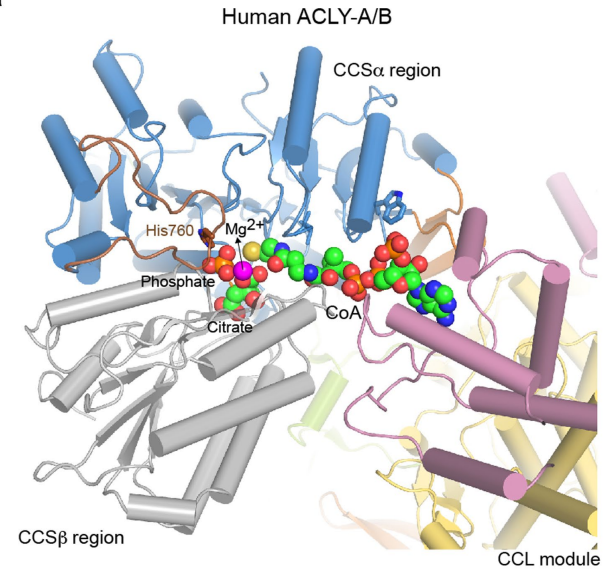

c

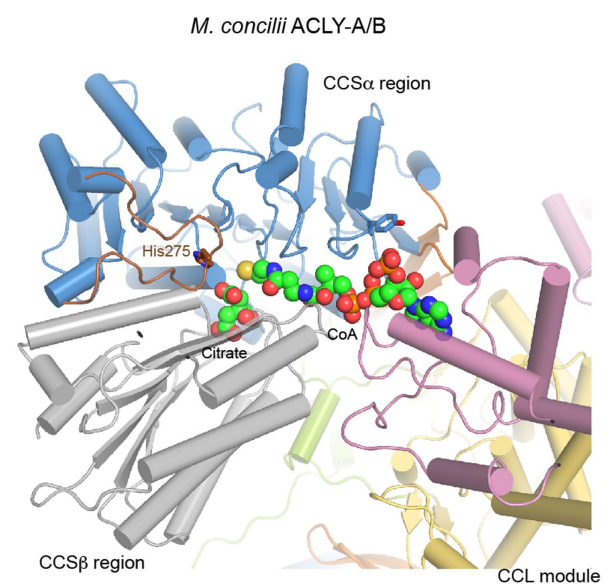

e

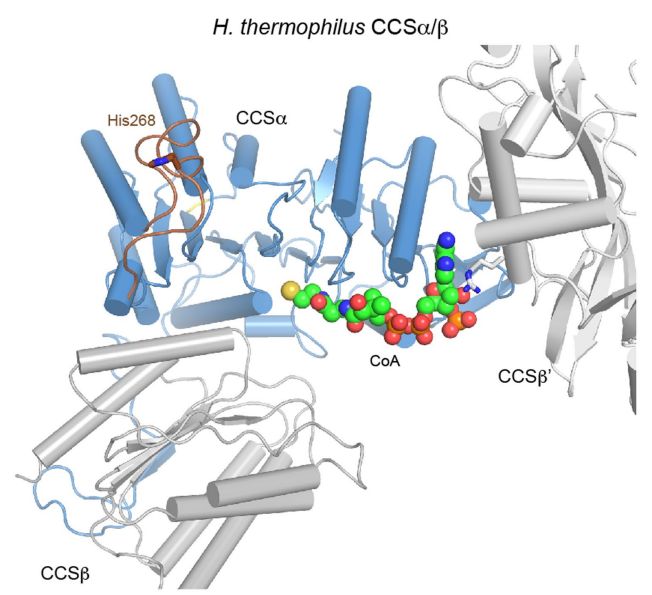

Extended Data Fig. 2 CoA-binding modes in the CCS-module of ACLY and related CCS. a, View on the CoA-binding mode in hACLY-A/B crystal structures. b, Detail of the CoA-binding mode at the interface between the CCL and CCS modules. The so-called power helices in the CCS module are indicated. Dashed lines represent polar interactions. c, View on the CoA-binding mode in the crystal structure for ACLY-A/B from M. concilii. d, View on the CoA-binding modes in

b

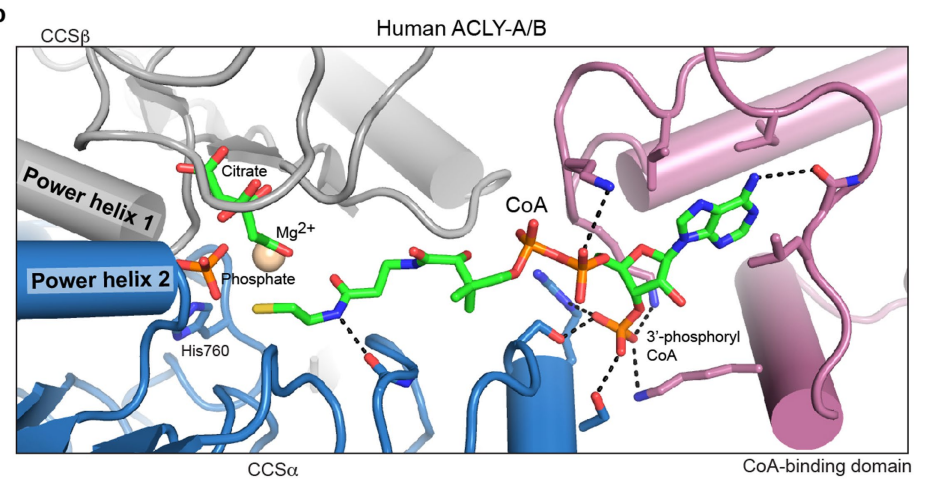

d
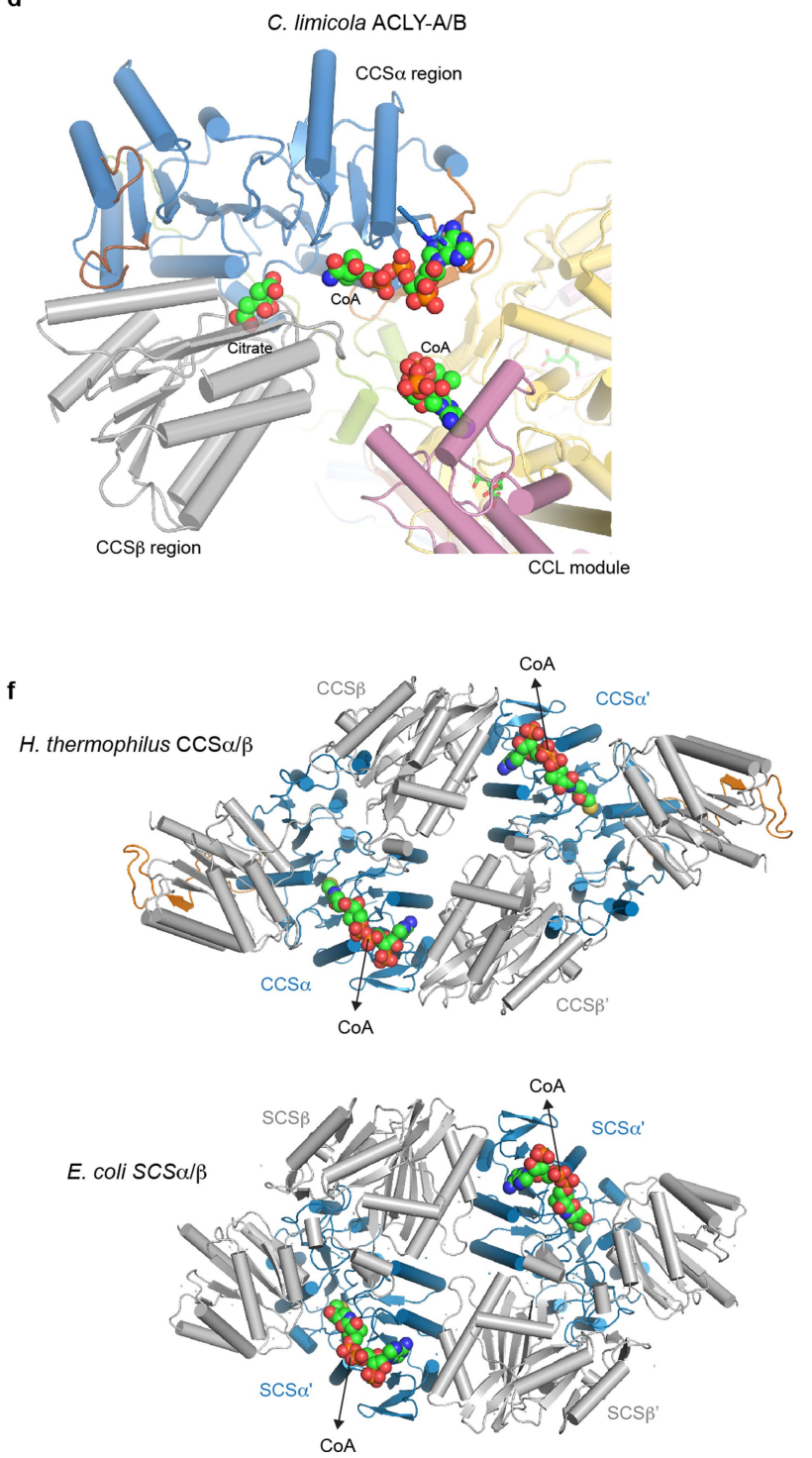

the crystal structure for ACLY-A/B from C. limicola. In this structure, the phosphopantheine tails of the CoA-molecules were partly disordered. e, View on the CoA-binding mode in CCS $\alpha / \beta$ from $H$. thermophilus. f, Cartoon representation of CCS $\alpha / \beta$ from $H$. thermophilus and succinylCoA synthetase $\alpha / \beta$ (SCS $\alpha / \beta)$ from $E$. coli, both in complex with CoA. $\alpha$-subunits are coloured in blue and $\beta$-subunits in grey. The $\mathrm{C}$-terminal tail extending from $\operatorname{CCS} \alpha$ to $\operatorname{CCS} \beta$ is in orange. 
$a$

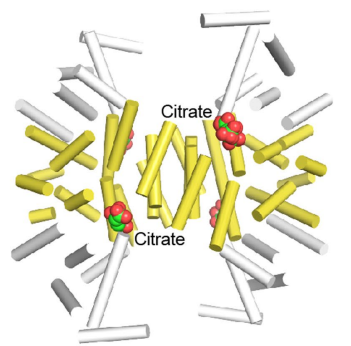

d
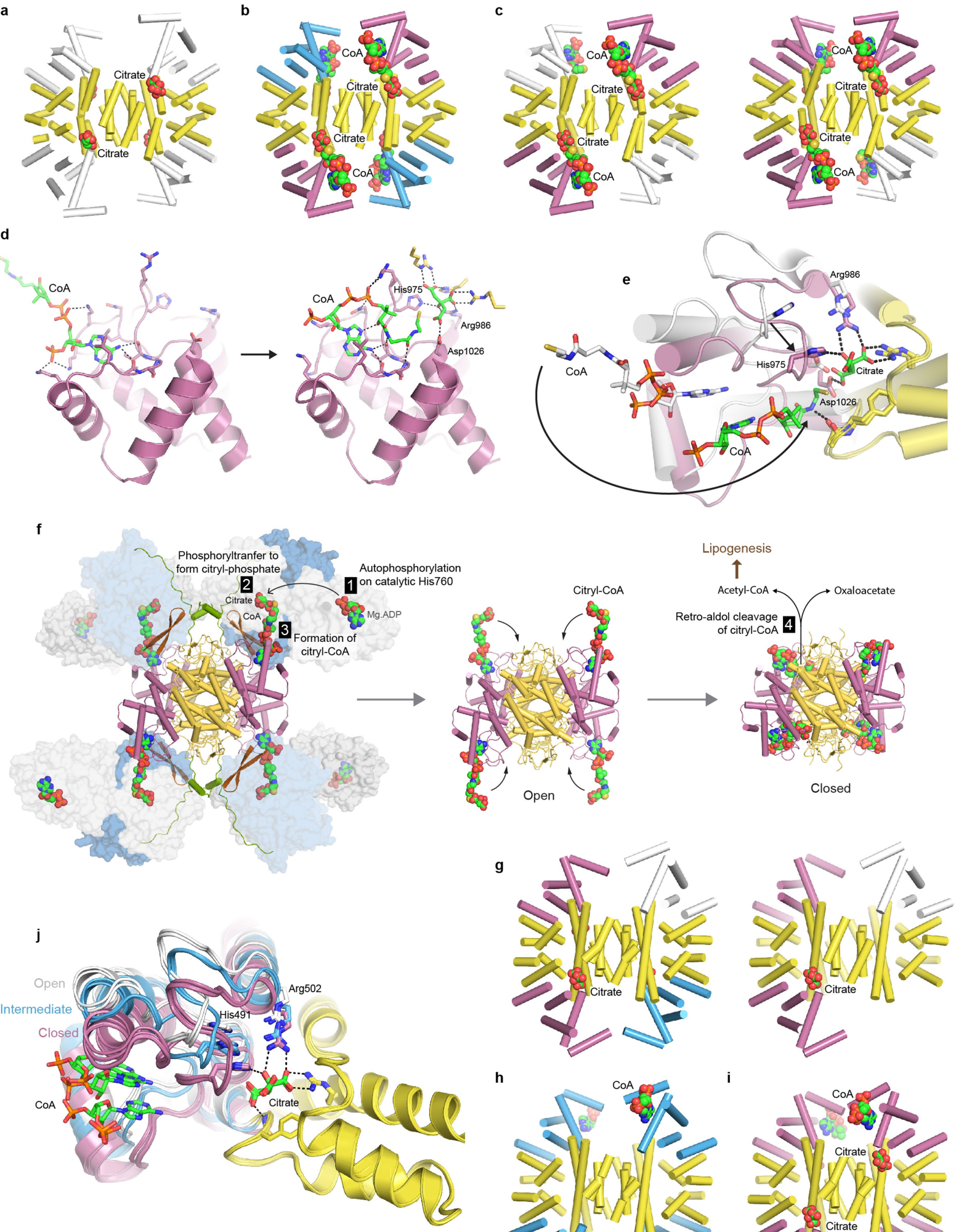

h
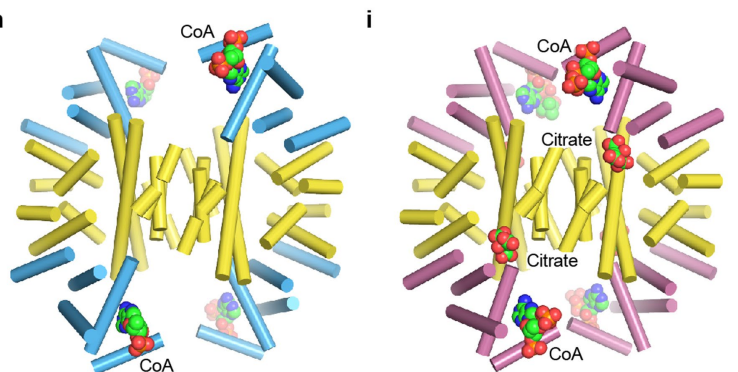

Extended Data Fig. 3 | See next page for caption. 


\section{RESEARCH LETTER}

Extended Data Fig. 3 | Structural plasticity in the CCL modules of human and C. limicola ACLY. a, Crystal structure of the CCL module of hACLY in space group $P 22_{1} 2_{1} 2_{1}$, in complex with citrate. $\mathbf{b}$, Crystal structure of the CCL module of hACLY in space group C $222_{1}$, in complex with citrate and CoA. c, Crystal structure of the CCL module of hACLY in space group $P 2_{1}$, in complex with citrate and CoA. This crystal form contained two tetramers in the crystallographic asymmetric unit (asu). In a-c, CoA-binding domains are coloured according to the structural state of the CCL active site: open (white), intermediate (blue) and closed (magenta), and substrates are shown as coloured spheres. d, Binding mode of CoA as seen in the hACLY-A/B crystal structure (left) compared to CoA binding in a closed CCL module protomer (right). Substrates are shown as coloured sticks and dashed lines indicate polar interactions. e, A CCL module protomer in the open state as seen in the hACLY-A/B structure (white CoA-binding domain) overlaid with a protomer in the closed state (magenta CoA-binding domain). The latter was extracted from a crystal structure for the isolated CCL module of hACLY (c). Arrows indicate structural transitions. f, Reaction itinerary in human ACLY. g, Crystal structure for the CCL module of C. limicola ACLY in space group $P 2{ }_{1}$, in complex with citrate. This crystal form contained two tetramers in the asu. In the second tetramer (right), one of the CoA-binding domains was not modelled owing to disorder. $\mathbf{h}$, Crystal structure for the CCL module of $C$. limicola ACLY in space group $P 3_{1} 21$, in complex with CoA. i, CCL module of C. limicola ACLY as observed in the C. limicola ACLY crystal structure. $\mathbf{j}$, Overlay of $C$. limicola CCL module protomers coloured according to the structural state of their active site: open (white), intermediate (blue) and closed (magenta). 

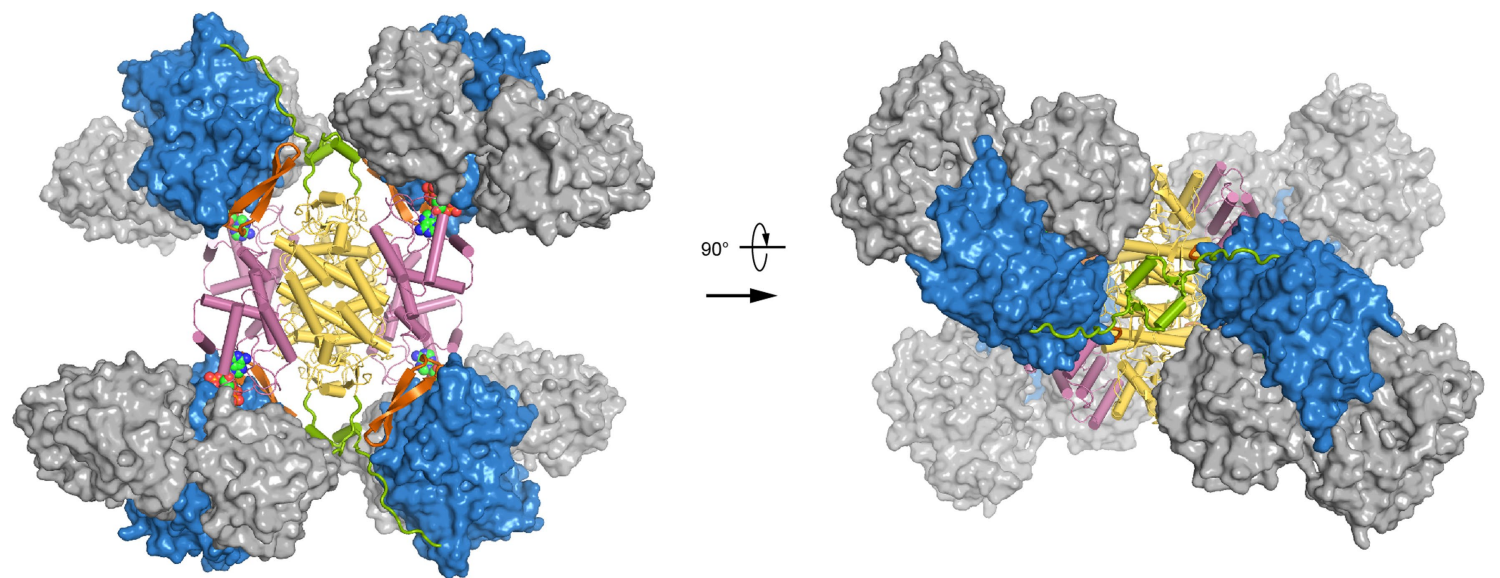

Human ACLY-A/B
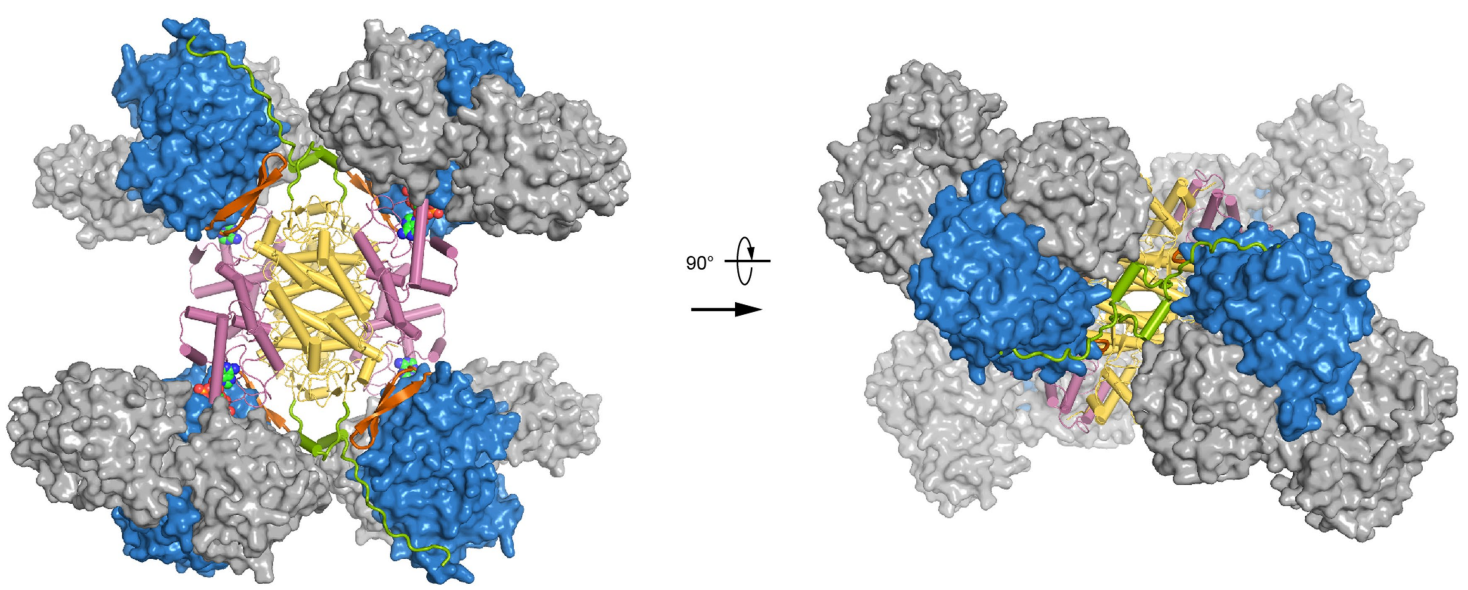

Methanosaeta concilii ACLY-A/B
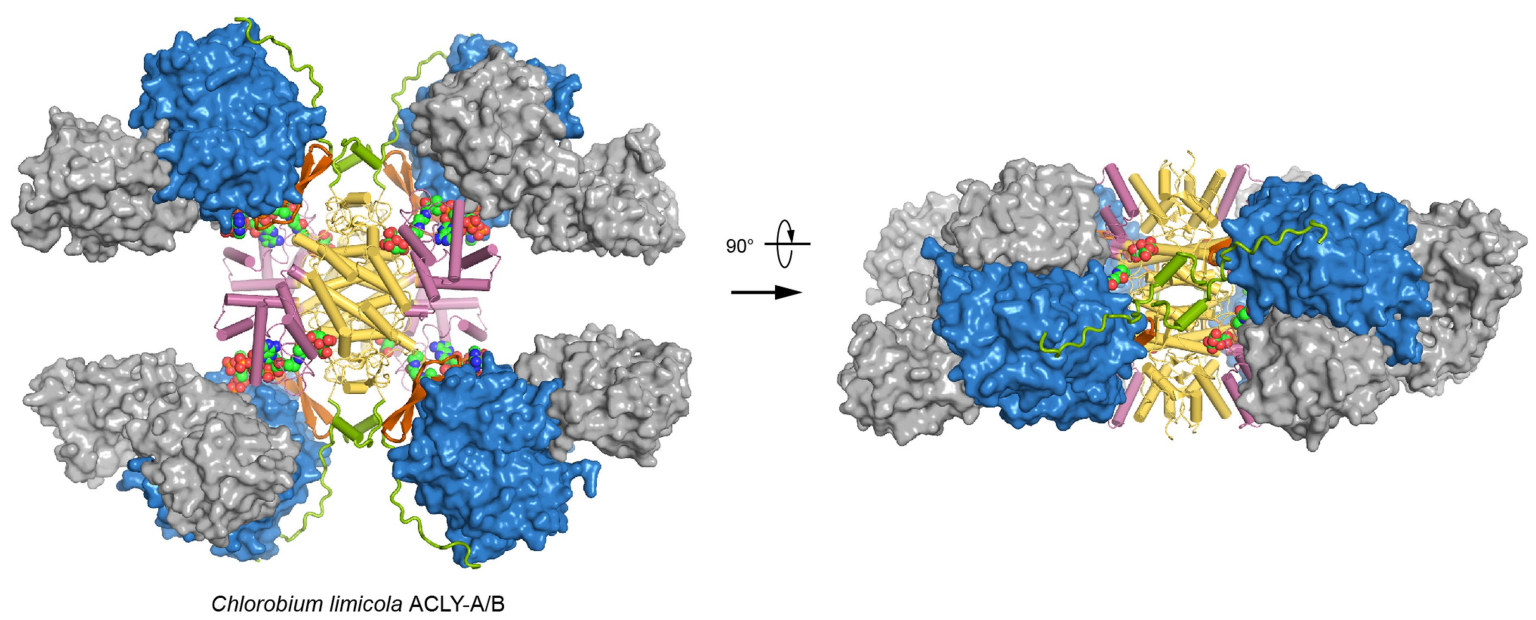

Extended Data Fig. 4 | ACLY structures across different domains of life. Cartoon representations of the crystal structures of human ACLY-A/B (top), M. concilii ACLY-A/B (middle) and C. limicola ACLY-A/B (bottom).
The CCS modules are shown in surface mode. Distinct structural regions are coloured according to the colouring scheme in Fig. 1a. 
a

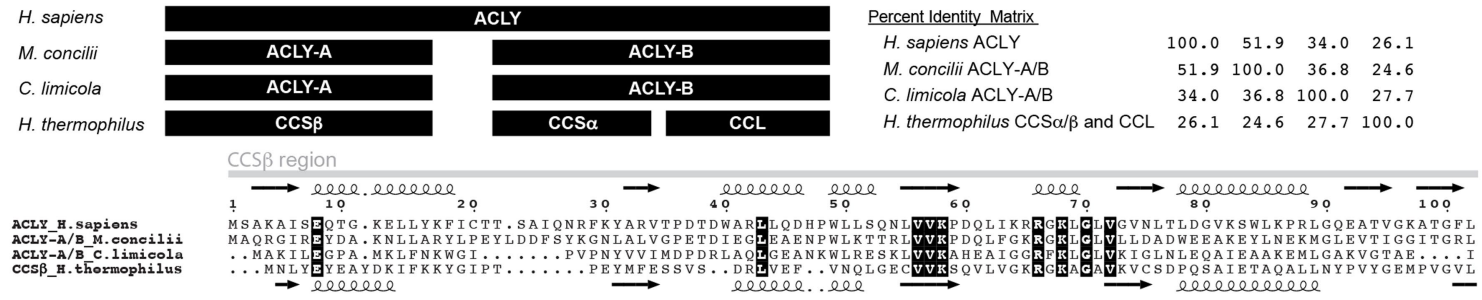

ACLY H. Sapions
ACLY-A/B_M. concili

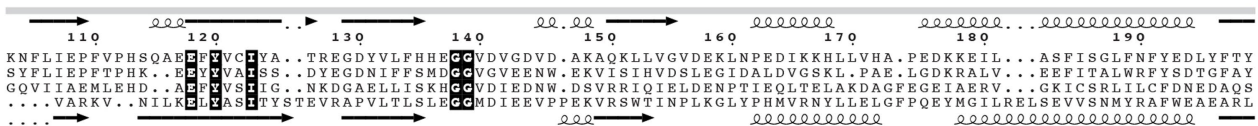
ACLY-A/BC. 1imico
$\operatorname{CCS} \beta$ B.thormophil

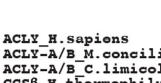

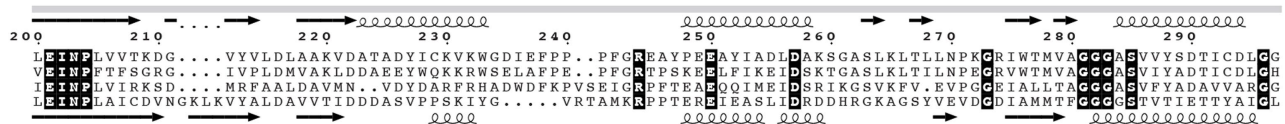

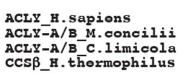

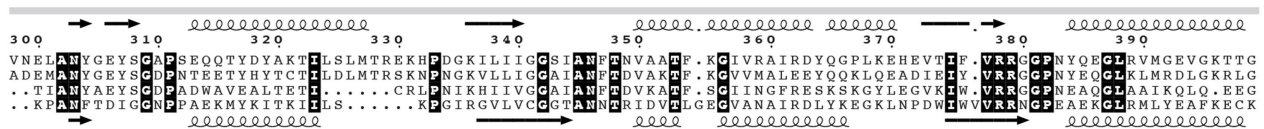

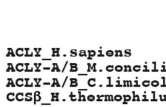

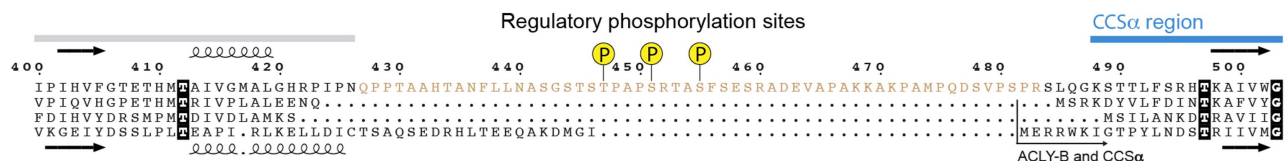

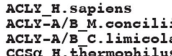

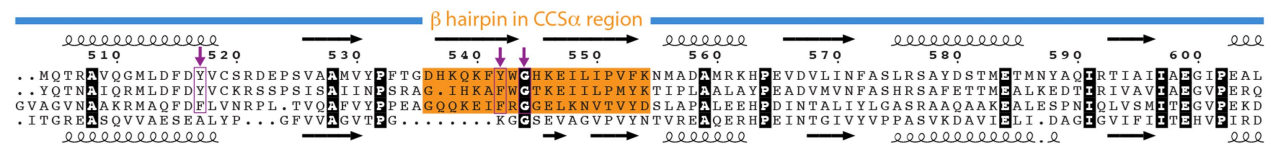
ACLY_H.sapions
ACLY-A/B M. concilii
ACLY-A/B-C.1imicola
CCSO_B.thormophilus

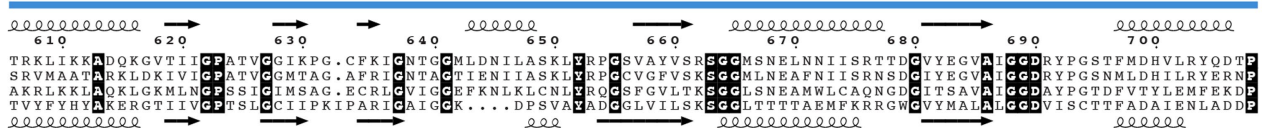
ACLY H. Sapiens
ACLY-A/B-M.coneilii
ACLY-A/B-C.1imicola
CCSC

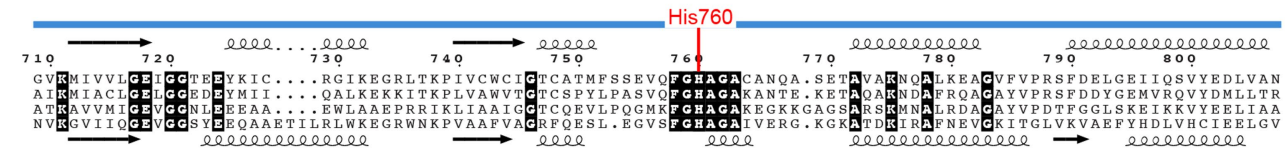

ACLY_H.sapions
ACLY-A/B_M. coneilii
ACLY-A/B-C.1imicola
CCL_H.thormophilus

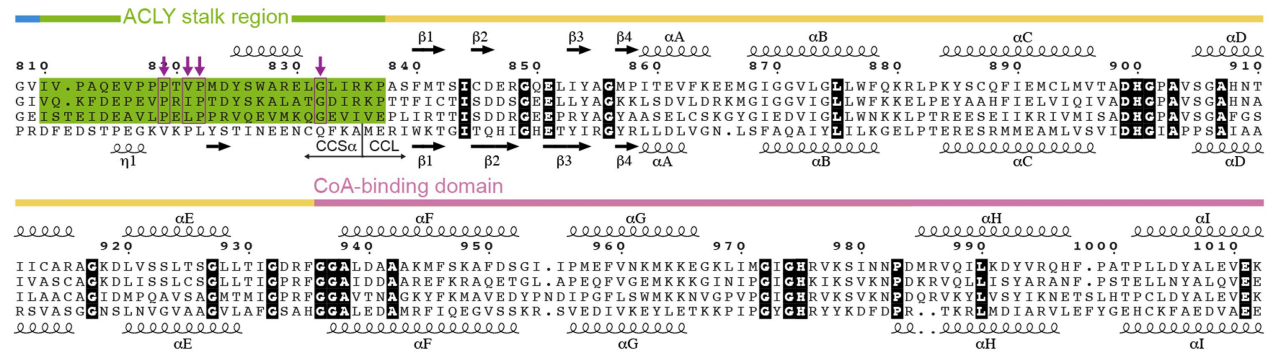

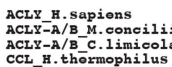
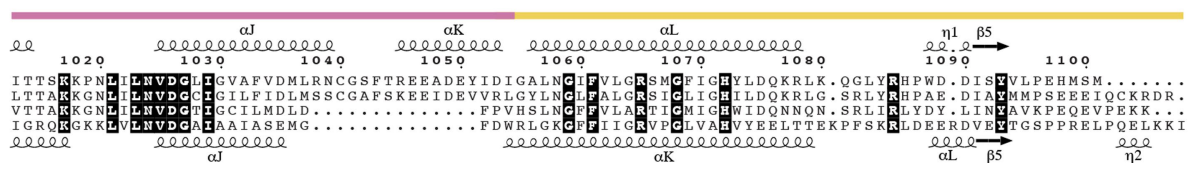

Extended Data Fig. 5 | Sequence alignment of ACLY, CCS and CCL. a, Homology relationships between the different enzymes for which crystal structures were determined in this manuscript. b. Sequence alignment for ACLY, CCL and CCS according to the scheme in a. Top secondary structure elements correspond to hACLY, bottom secondary structure elements correspond to CCS $\alpha / \beta$ and CCL from $H$. thermophilus. Strictly conserved residues are white against a black background. $\operatorname{CCS} \alpha, \operatorname{CCS} \beta$ and CCL homology regions and CoA-binding domain are indicated by a coloured bar on top of the alignment. The CCS $\alpha \beta$-hairpin (orange) and CCL stalk (green) regions in ACLY enzymes are highlighted. Conserved residues at the ACLY two-helix pivot are indicated with a purple arrow. Regulatory phosphorylation sites in the linker region (brown) that connects the ancestral ACLY-A and ACLY-B parts in hACLY are indicated by a letter $\mathrm{P}$ in yellow circles. 
a

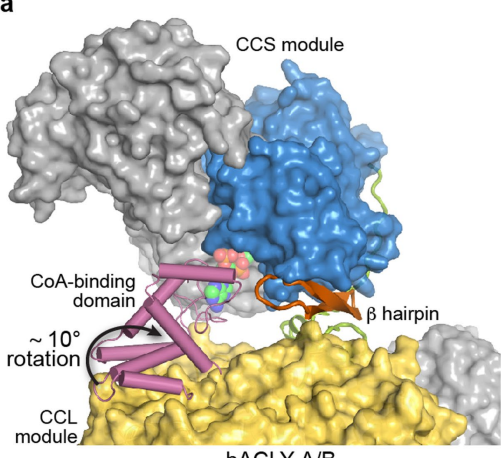

b

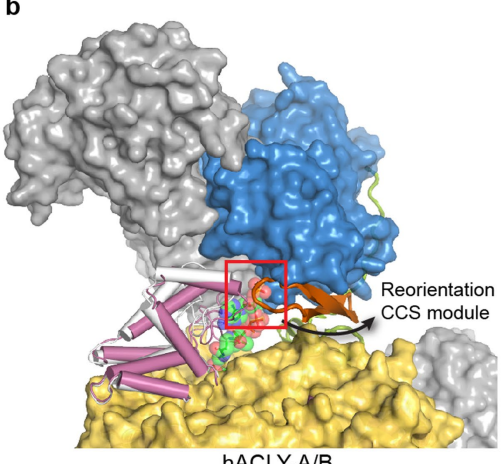

c

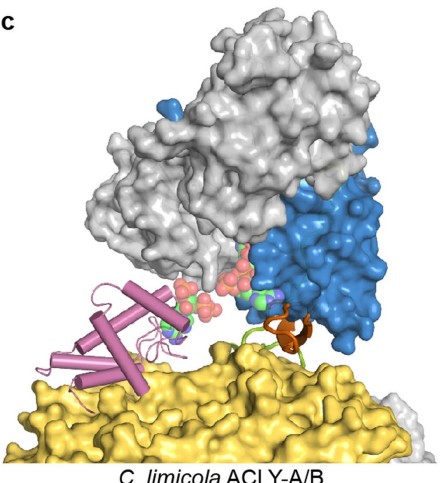

d

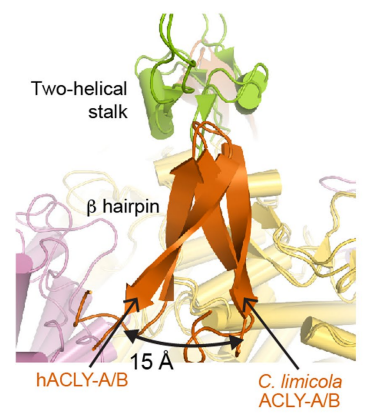

g

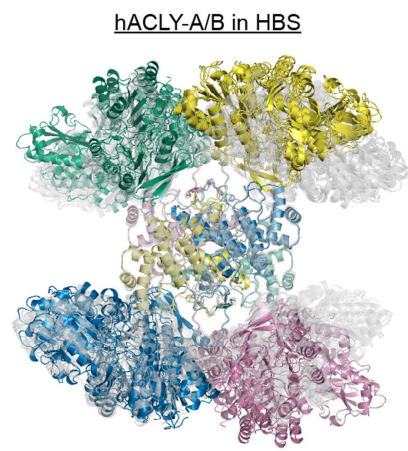

$0^{\circ} \frac{\mathbb{t}}{t} \downarrow$
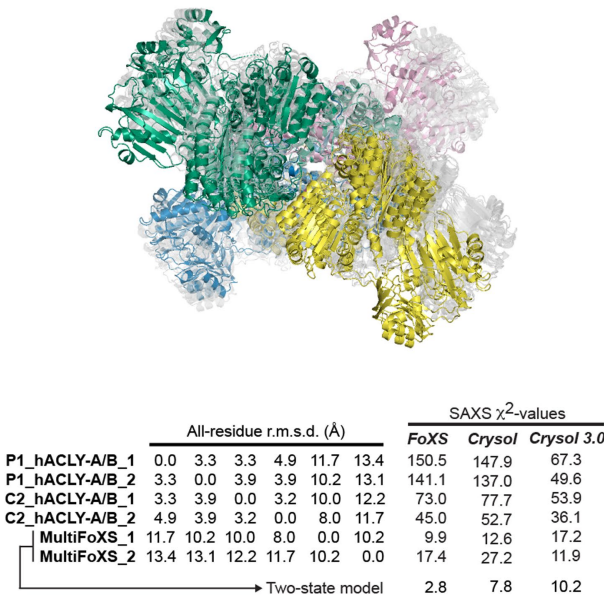

Extended Data Fig. 6 | See next page for caption.

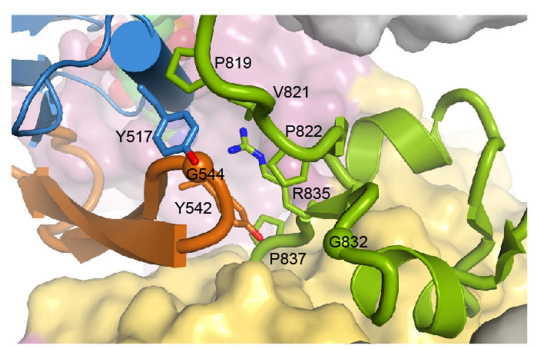

hACLY-A/B

h

hACLY-A/B in HBS + citrate and CoA

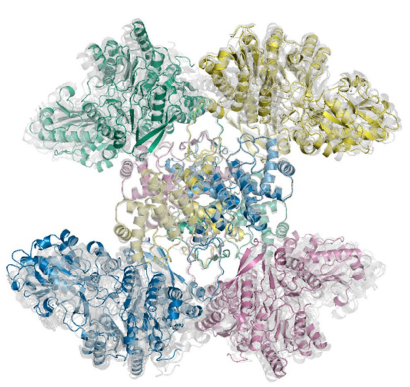

${ }_{90}^{\circ} \mathfrak{t}$

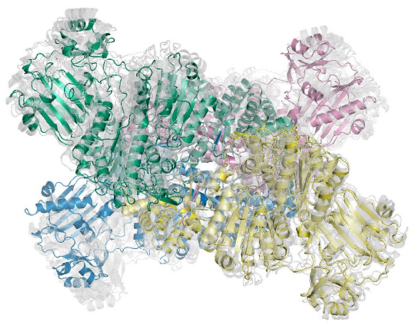

f

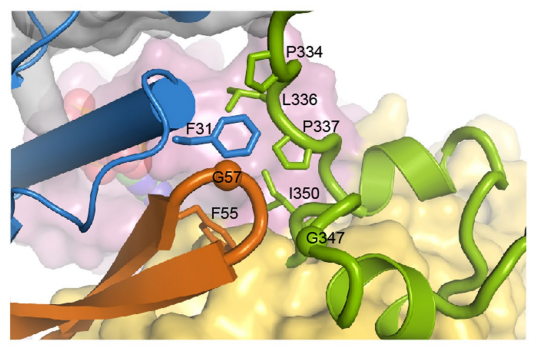

C. limicola ACLY-A/B

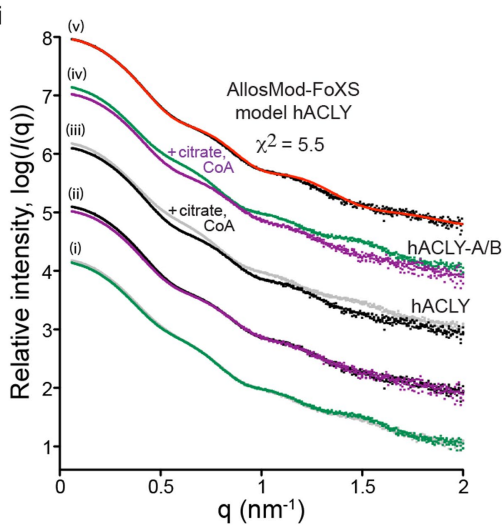

j hACLY in HBS + citrate and CoA
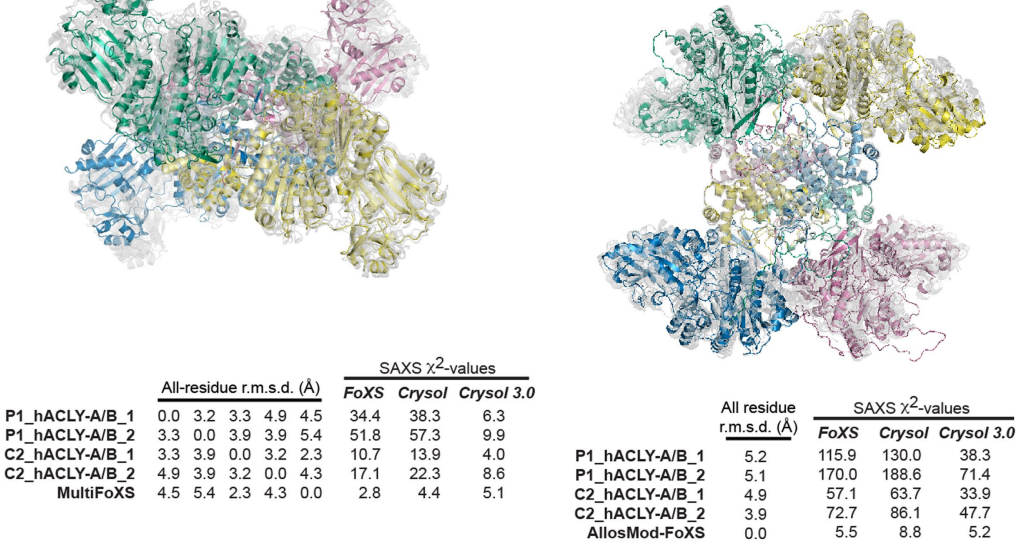
Extended Data Fig. 6 | Conformational switching of ACLY during catalysis. a, View of the interaction between the CCS and CCL modules in a representative hACLY-A/B crystal structure (space group $P 1$ ), with the $\operatorname{CCS} \alpha \beta$-hairpin (orange) and CoA-binding domain (pink) in cartoon mode. Bound CoA is shown as coloured spheres. b, Overlay of a human CCL protomer in the closed state (pink CoA-binding domain) with the crystal structure of hACLY-A/B as in a (white CoA-binding domain). The resulting clash between the CoA-binding domain (with bound CoAmolecule) and the $\beta$-hairpin is indicated by a red box. c, View of the interaction between the CCS and CCL modules in the crystal structure of $C$. limicola ACLY-A/B, with the CCS $\alpha \beta$-hairpin (orange) and CoAbinding domain (pink) in cartoon mode. $\mathbf{d}$, Zoomed-in view of the stalk region in the crystal structures of hACLY-A/B and C. limicola ACLY-A/B based on the superposition of the helical core of the CCL modules.

e, $\mathbf{f}$, Interactions at the stalk region and $\beta$-hairpin as observed in the crystal structures of hACLY-A/B and C. limicola ACLY-A/B. g, Two-state rigidbody SAXS model for apo-hACLY-A/B (MultiFoXS, $\chi^{2}=2.8$ ) overlaid with the hACLY-A/B crystal structures in space groups $P 1$ and $C 2$ (grey). h, Single-state rigid-body SAXS model for hACLY-A/B (MultiFoXS, $\left.\chi^{2}=2.8\right)$ in the presence of both citrate and CoA overlaid with the hACLY-A/B crystal structures in space groups $P 1$ and $C 2$ (grey).

i, Comparison between in-solution SAXS scattering profiles measured from linker-deleted hACLY-A/B and full-length hACLY. (i) Profiles recorded from hACLY-A/B (green) and hACLY (grey) in HBS buffer; (ii) profiles recorded from hACLY-A/B (purple) and hACLY (black) in HBS buffer supplemented with citrate and CoA; (iii) profiles recorded from hACLY in HBS buffer (grey) and HBS buffer supplemented with both citrate and CoA (black); (iv) profiles recorded from hACLY-A/B in HBS buffer (green) and HBS buffer supplemented with both citrate and CoA (purple); and (v) fit of the theoretical scattering profile (red) calculated from an AllosMod-FoXS model for hACLY (as shown in $\mathbf{j}$ ) to the experimental scattering profile recorded in the presence of citrate and CoA (black). $\mathbf{j}$, AllosMod-FoXS SAXS model for hACLY in HBS buffer supplemented with citrate and CoA, overlaid with the hACLY-A/B crystal structures in space groups $P 1$ and $C 2$ (grey). In $\mathbf{g}, \mathbf{h}$ and $\mathbf{j}$, the bottom numeric table presents an all-residue (C $\alpha$ ) r.m.s.d. matrix for the hACLY$\mathrm{A} / \mathrm{B}$ crystal structures and presented SAXS models, and for each crystal structure and model the calculated fit ( $\chi^{2}$ value) against the recorded SAXS data are shown as calculated by FoXS, Crysol and Crysol 3.0. P1hACLY-A/B_1 and P1-hACLY-A/B_2 denote structures for hACLY-A/B extracted from the $P 1$ crystal form; C2-hACLY-A/B_1 and C2-hACLY$\mathrm{A} / \mathrm{B} \_2$ denote structures for hACLY-A/B extracted from the $C 2$ crystal form. 


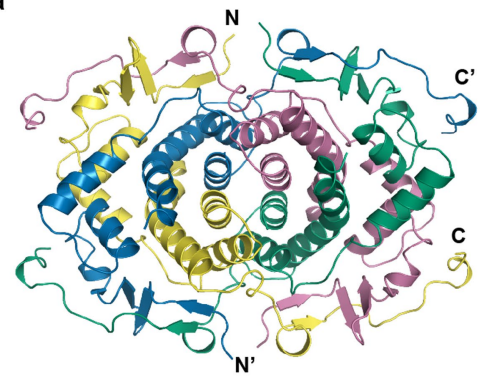

CCL H. thermophilus

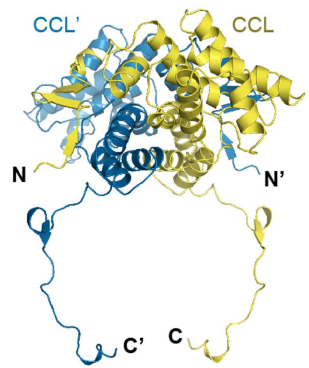

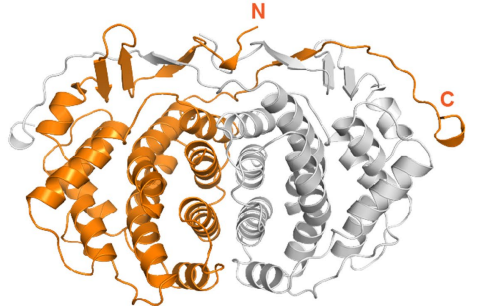

CS P. furiosis

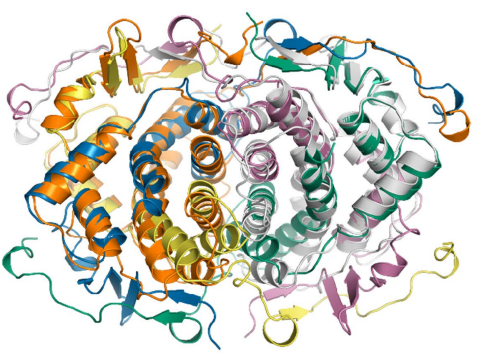

Overlay CLL and CS

r.m.s.d. $=1.5 \AA$ for 399 aligned C $\alpha$ 's

g
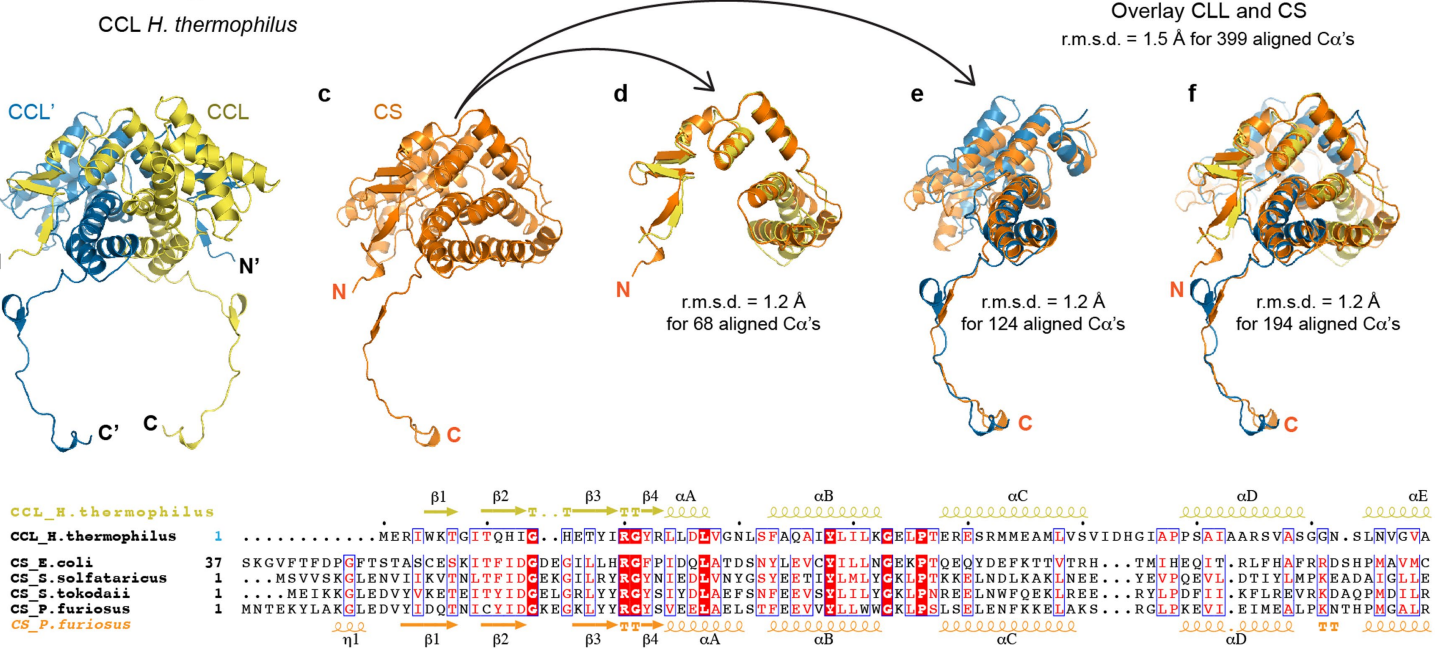

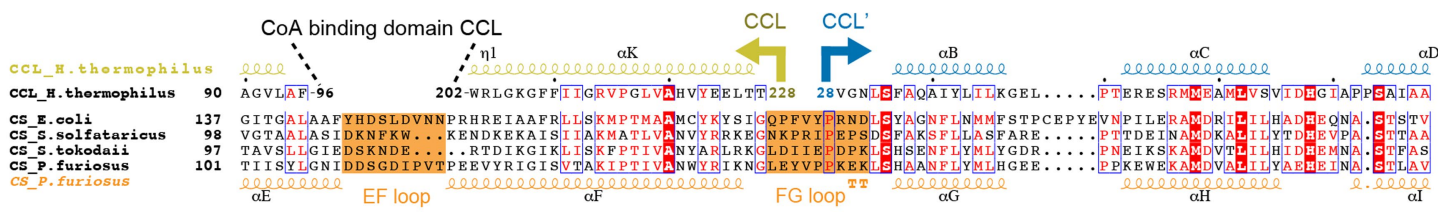

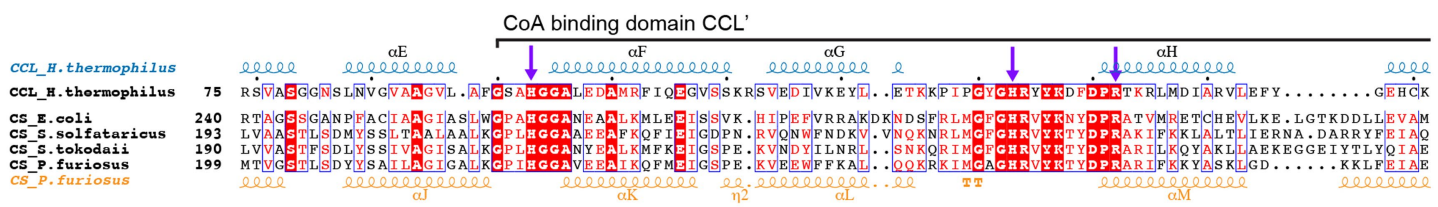

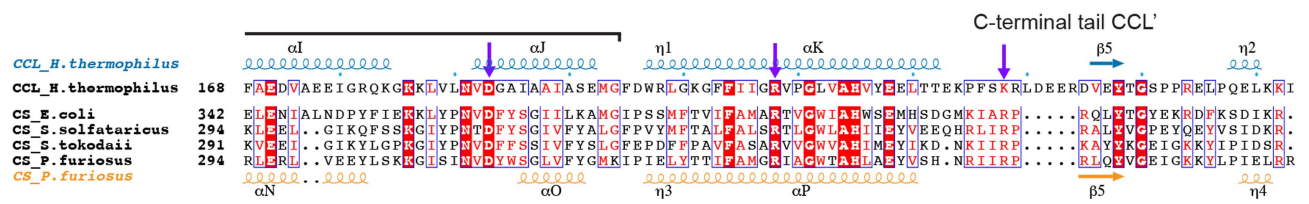

h

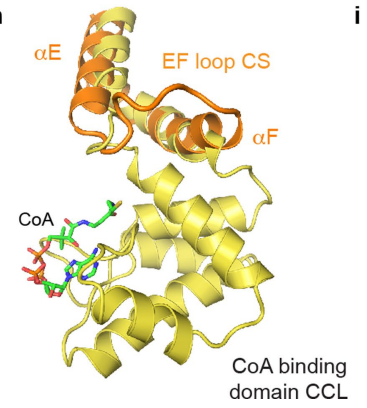

Extended Data Fig. 7 | Citrate synthase evolved from an ancestral CCL module. a, Side-by-side comparison and overlay of the helical bundle cores of $H$. thermophilus CCL and P. furiosus CS (PDB accession 1AJ8). b, Two adjacent CCL protomers (CCL and CCL') extracted from the H. thermophilus CCL tetramer. c, A CS protomer extracted from P. furiosus CS. d, CCL without its CoA-binding domain (residues 2-100 and 204-231) aligned with the N-terminal half of CS (residues 6-143). e, CCL' (residues 30-256) aligned with the C-terminal half of CS (residues
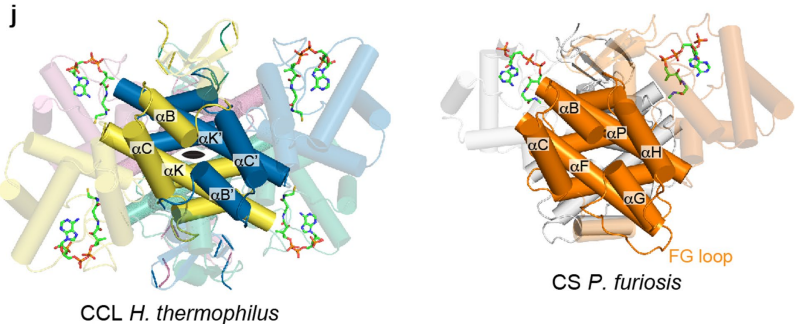

CS P. furiosis

154-376). f, CCL and CCL' aligned with the CS protomer. g, Sequence alignment between CCL and CCL' and CS sequences. Top secondary structure elements correspond to $H$. thermophilus CCL and CCL', bottom secondary structures correspond to $P$. furiosus CS. The active site residues of CS are indicated by a purple arrow. $\mathbf{h}, \mathbf{i}$, Details of the overlay between CCL and CCL' and the CS protomer. $\mathbf{j}$, Side view of the CCL module and CS highlighting the pseudo-two-fold symmetry in the CS protomer. CoA is shown by sticks. 
a



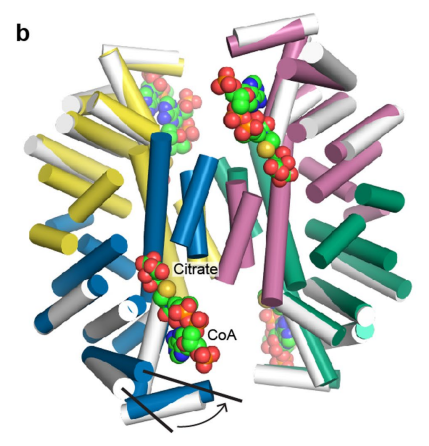

CCL module hACLY

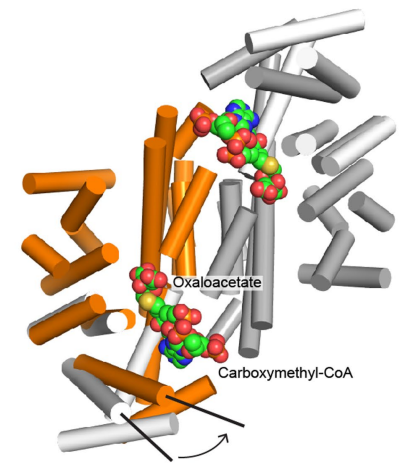

Chicken CS

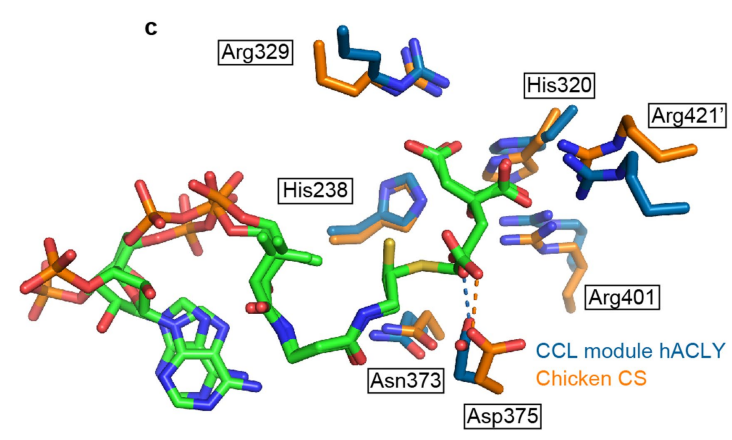

d

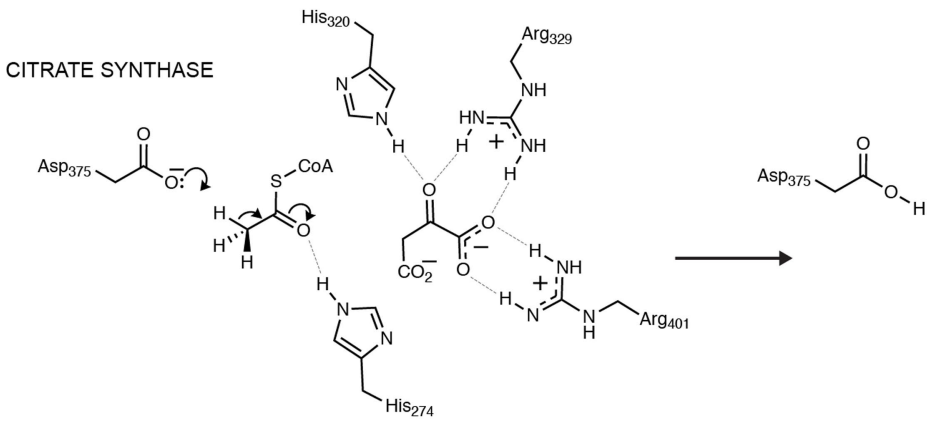

Deprotonation acetyl-CoA

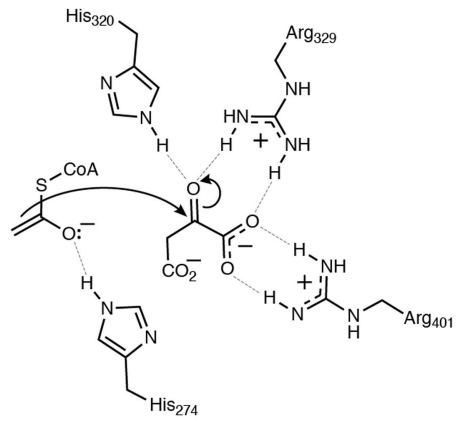

Aldol condensation forming citryl-CoA
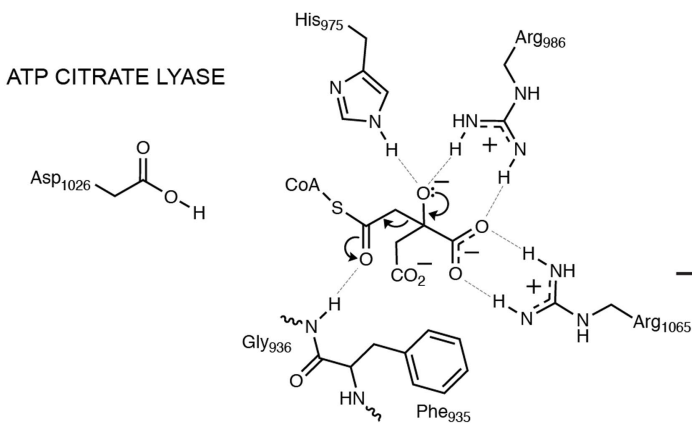

Retroaldol cleavage citryl-CoA

Extended Data Fig. 8 Homology between ACLY and citrate synthase. a, Sequence alignment between the C-terminal regions of ACLY, CCL and CS. Active-site residues are highlighted according to the numbering scheme in chicken CS. His274, highlighted in yellow, is not conserved in ACLY sequences. b, Comparison between crystal structures for the CCL module of hACLY and chicken CS (PDB accessions 5CSC and 5CTS) in open and closed states. For clarity, only the helical secondary structure elements are shown. Bound substrates are shown as coloured spheres. c, Overlay of the CCL active site of hACLY (blue) in complex with

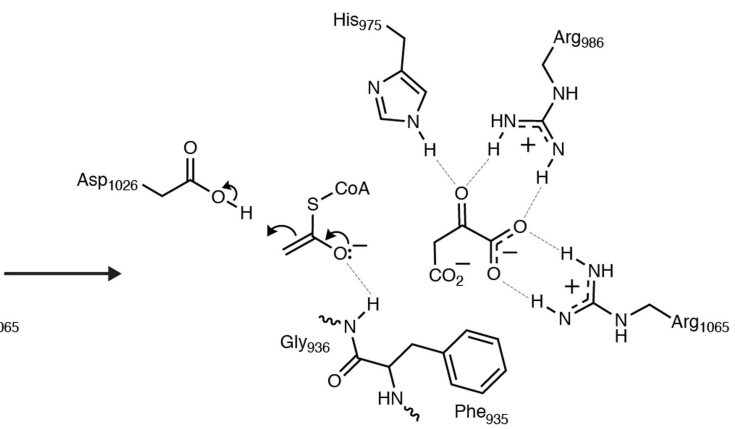

Protonation acetyl-CoA enolate citrate and CoA, with the active site of chicken CS (orange) in complex with oxaloacetate and carboxymethyl-CoA (PDB accession 5CTS). The interaction between the carboxylate group of hACLY(Asp1026) and citrate, and the interaction between the carboxylate group of Asp375 of CS and carboxymethyl-CoA are indicated. d, By analogy to the aldol condensation of acetyl-CoA and oxaloacetate to citryl-CoA as catalysed by CS (top), citryl-CoA may undergo retro-aldol cleavage catalysed by ACLY as indicated by the chemical reaction arrows (bottom). Dashed lines indicate polar interactions. 
cleaned dataset: 27293 particles

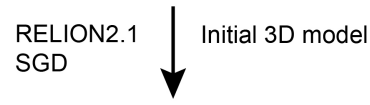

D2

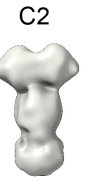

C1

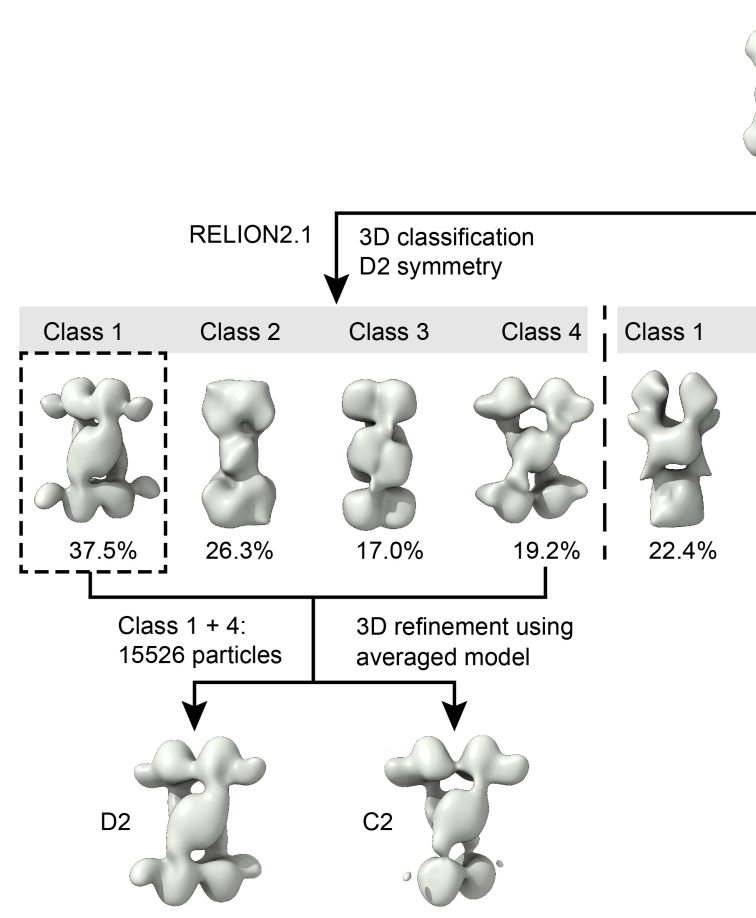

Extended Data Fig. 9 | Flowchart showing negative-stain electron microscopy data processing for human ACLY. Starting from a final dataset of 27,293 particles, initial models were made using the stochastic gradient descent method in RELION2.1, applying C1, C2 or D2 symmetry. Subsequent 3D classification was performed using the C1, C2 or D2 starting models as an input, again applying C1, C2 or D2 symmetry, respectively. 3D classification using $\mathrm{C} 1$ and $\mathrm{C} 2$ symmetry clearly shows
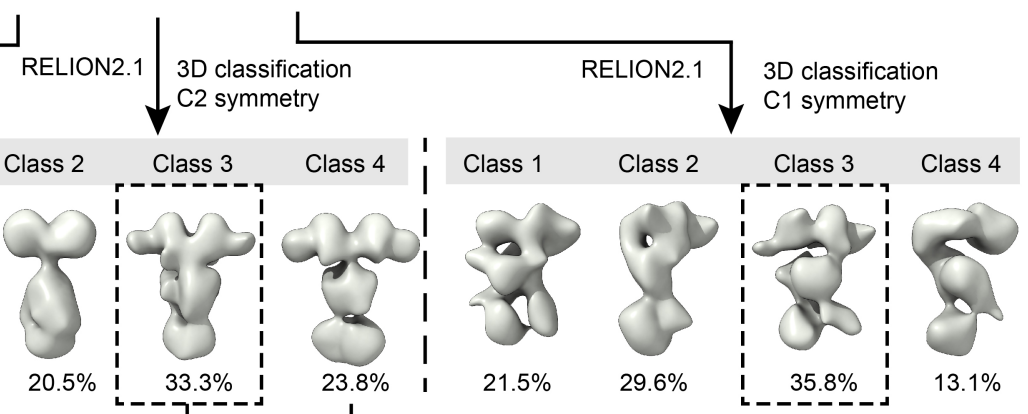

well-defined CCS modules in one-half of the hACLY molecule (C1: class 3, $\mathrm{C} 2$ : class 3 and 4). Although 3D classification using D2 symmetry results in two classes displaying all four CCS modules (class 1 and 4), subsequent $3 \mathrm{D}$ refinement in $\mathrm{C} 2$ using an averaged map of these two classes resulted in a disappearance of two CCS modules in the lower half of hACLY, pointing to flexibility of the peripheral domains of hACLY. 
Extended Data Table 1 | Crystallographic data and refinement statistics

\begin{tabular}{|c|c|c|c|c|c|c|c|c|c|c|c|c|}
\hline \multicolumn{5}{|c|}{ ACLY-A/B } & \multicolumn{6}{|c|}{ CCL module of ACLY } & $\mathrm{CCL}$ & \multirow{2}{*}{$\begin{array}{c}\text { CCS } \\
\text { H. thermophilu. }\end{array}$} \\
\hline Source organism & H. sapiens & H. sapiens & M. concilii & C. limicola & H. sapiens & H. sapiens & H. sapiens & C. limicola & C. limicola & C. limicola & H. thermophilus & \\
\hline $\begin{array}{l}\text { Crystallization } \\
\text { conditions }\end{array}$ & $\begin{array}{c}13.5 \% \mathrm{PEG}_{3350} ; \\
0.2 \mathrm{M} \mathrm{Na} \mathrm{HPO}_{4} \\
\mathrm{pH} 8.15\end{array}$ & $\begin{array}{c}17 \% \mathrm{PEG}_{3350} \\
0.2 \mathrm{M} \mathrm{Na}_{2} \mathrm{HPO}_{4} \\
\mathrm{pH} 8.15\end{array}$ & $\begin{array}{c}14 \% \mathrm{PEG}_{3350} \\
4 \% \text { tacsimate } \\
\text { pH } 8.0\end{array}$ & $\begin{array}{c}24 \% \text { PEG } \\
\text { Smear High; } \\
0.1 \text { M Tris } \\
\text { pH } 8.0\end{array}$ & $\begin{array}{c}0.2 \mathrm{M} \mathrm{Na}_{2} \mathrm{SO} 4 \\
\mathrm{pH} 6.7 \\
20 \% \mathrm{PEG}_{3350}\end{array}$ & $\begin{array}{c}21 \% \mathrm{PEG}_{8000} ; \\
0.1 \mathrm{M} \mathrm{HEPES} \\
\mathrm{pH} \mathrm{7.0;} \\
0.15 \mathrm{M}\left(\mathrm{NH}_{4}\right)_{2} \mathrm{SO}_{4}\end{array}$ & $\begin{array}{c}0.1 \mathrm{M} \\
\mathrm{Na} \mathrm{KH}_{2} \mathrm{PO}_{4} \\
\mathrm{pH} 5.5 \\
0.1 \mathrm{M} \mathrm{RbCl} \\
0.1 \mathrm{M} \mathrm{Na}-\mathrm{citrate} \\
\mathrm{pH} 5.5 \\
25 \% \text { PEG } \\
\text { Smear Medium }\end{array}$ & $\begin{array}{l}1.6 \mathrm{M} \mathrm{Li}_{2} \mathrm{SO}_{4} \\
0.1 \mathrm{M} \mathrm{Tris} \\
\mathrm{pH} 8.0\end{array}$ & $\begin{array}{c}22 \% \text { PEG Smear } \\
\text { Broad; } \\
0.1 \mathrm{M} \mathrm{MES} \\
\text { pH } 6.5\end{array}$ & $\begin{array}{c}0.2 \mathrm{M} \mathrm{CaCl}_{2} \\
\mathrm{pH} \mathrm{5.1;} \\
20 \% \mathrm{PEG}_{3350}\end{array}$ & $\begin{array}{c}45 \% \text { MPD; } \\
0.1 \mathrm{M} \mathrm{HEPES} \\
\text { pH } 7.5 ; \\
0.2 \mathrm{M} \mathrm{NH}_{4} \mathrm{Ac}\end{array}$ & $\begin{array}{l}18 \% \mathrm{PEG}_{\text {mooo }} ; \\
20 \% \text { propanol; } \\
0.1 \mathrm{M} \text { sodium } \\
\text { citrate pH } 5.6\end{array}$ \\
\hline $\begin{array}{l}\text { Protein } \\
\text { concentration }\end{array}$ & $8 \mathrm{mg} / \mathrm{ml}$ & $14 \mathrm{mg} / \mathrm{ml}$ & $7 \mathrm{mg} / \mathrm{ml}$ & $12 \mathrm{mg} / \mathrm{ml}$ & $18 \mathrm{mg} / \mathrm{ml}$ & $7 \mathrm{mg} / \mathrm{ml}$ & $7 \mathrm{mg} / \mathrm{ml}$ & $13 \mathrm{mg} / \mathrm{ml}$ & $12 \mathrm{mg} / \mathrm{ml}$ & $12 \mathrm{mg} / \mathrm{ml}$ & $7 \mathrm{mg} / \mathrm{ml}$ & $11 \mathrm{mg} / \mathrm{ml}$ \\
\hline Cryoprotectant & $25 \%$ glycerol & $25 \%$ glycerol & $22 \% \mathrm{PEG}_{400}$ & $22 \%$ PEG 400 & $25 \% \mathrm{PEG} 400$ & $25 \% \mathrm{PEG}_{400}$ & $25 \% \mathrm{PEG}_{400}$ & $20 \%$ glycerol & $25 \%$ PEG 400 & $30 \%$ PEG 400 & $49 \%$ MPD & $22 \% \mathrm{PEG}_{400}$ \\
\hline Ligands & $\begin{array}{c}\text { Citrate, } \\
\text { CoA, } \\
\mathrm{Mg} . \mathrm{ADP} \\
\mathrm{H}_{2} \mathrm{PO}_{4}^{-}\end{array}$ & $\begin{array}{c}\text { Citrate, } \\
\text { CoA, } \\
\text { Mg.ADP, } \\
\mathrm{H}_{2} \mathrm{PO}_{4}^{-}\end{array}$ & $\begin{array}{l}\text { Citrate, } \\
\text { CoA }\end{array}$ & $\begin{array}{l}\text { Citrate, } \\
\text { CoA }\end{array}$ & Citrate & $\begin{array}{l}\text { Citrate, } \\
\mathrm{CoA}\end{array}$ & $\begin{array}{l}\text { Citrate, } \\
\text { CoA }\end{array}$ & $\mathrm{CoA}$ & Citrate & $\begin{array}{c}\text { L-malate, } \\
\text { Acetyl-CoA }\end{array}$ & $\mathrm{CoA}$ & $\mathrm{CoA}$ \\
\hline \multicolumn{13}{|l|}{ Data collection } \\
\hline $\begin{array}{l}\text { X-ray source } \\
\text { (beamline) }\end{array}$ & $\begin{array}{l}\text { SLS } \\
\text { (PXI) }\end{array}$ & $\begin{array}{l}\text { PETRAIII } \\
\text { (P14) }\end{array}$ & $\begin{array}{c}\text { SLS } \\
\text { (PXIII) }\end{array}$ & $\begin{array}{c}\text { ESRF } \\
\text { (ID30-B) }\end{array}$ & $\begin{array}{l}\text { PETRAIII } \\
\text { (P14) }\end{array}$ & $\begin{array}{c}\text { SLS } \\
\text { (PXIII) }\end{array}$ & $\begin{array}{c}\text { SLS } \\
\text { (PXIII) }\end{array}$ & $\begin{array}{c}\text { SOLEIL } \\
\text { (Proxima 2A) }\end{array}$ & $\begin{array}{c}\text { ESRF } \\
\text { (ID23-1) }\end{array}$ & $\begin{array}{l}\text { PETRAIII } \\
\text { (P13) }\end{array}$ & $\begin{array}{c}\text { SLS } \\
\text { (PXIII) }\end{array}$ & $\begin{array}{c}\text { SLS } \\
\text { (PXIII) }\end{array}$ \\
\hline Wavelength $(\lambda)$ & 0.999977 & 0.9763 & 1.00003 & 0.97625 & 0.9762 & 1.00003 & 1.00003 & 0.980042 & 0.97242 & 0.9762 & 1.00003 & 1.00003 \\
\hline Space group & $P 1$ & $C 2$ & $P 21212$ & $P 21$ & $P 212121$ & $P 21$ & C2221 & $P 3121$ & $P 21$ & $P 21$ & $I 4122$ & $P 212121$ \\
\hline \multicolumn{13}{|l|}{ Cell dimensions } \\
\hline$a, b, c(\AA)$ & $\begin{array}{l}150.36 \\
154.01 \\
154.09\end{array}$ & $\begin{array}{l}214.28 \\
215.40 \\
158.42\end{array}$ & $\begin{array}{c}118.45 \\
275.02 \\
72.72\end{array}$ & $\begin{array}{l}101.67 \\
134.93 \\
177.71\end{array}$ & $\begin{array}{l}67.34 \\
123.89 \\
139.80\end{array}$ & $\begin{array}{l}67.71 \\
147.90 \\
113.69\end{array}$ & $\begin{array}{l}82.83 \\
111.19 \\
143.37\end{array}$ & $\begin{array}{c}110.03 \\
110.03 \\
92.75\end{array}$ & $\begin{array}{c}99.26 \\
106.41 \\
105.49\end{array}$ & $\begin{array}{l}102.73 \\
100.82 \\
105.01\end{array}$ & $\begin{array}{c}130.60 \\
130.60 \\
83.95\end{array}$ & $\begin{array}{l}118.50 \\
127.03 \\
143.80\end{array}$ \\
\hline$\alpha, \beta, \gamma\left({ }^{\circ}\right)$ & $\begin{array}{l}91.53 \\
110.04 \\
107.46\end{array}$ & $\begin{array}{l}90.00 \\
117.27 \\
90.00\end{array}$ & $\begin{array}{l}90.00 \\
90.00 \\
90.00\end{array}$ & $\begin{array}{c}90.00 \\
101.65 \\
90.00\end{array}$ & $\begin{array}{l}90.00 \\
90.00 \\
90.00\end{array}$ & $\begin{array}{c}90.00 \\
105.28 \\
90.00\end{array}$ & $\begin{array}{l}90.00 \\
90.00 \\
90.00\end{array}$ & $\begin{array}{l}90.00 \\
90.00 \\
120.00\end{array}$ & $\begin{array}{c}90.00 \\
102.11 \\
90.00\end{array}$ & $\begin{array}{c}90.00 \\
102.99 \\
90.00\end{array}$ & $\begin{array}{l}90.00 \\
90.00 \\
90.00\end{array}$ & $\begin{array}{l}90.00 \\
90.00 \\
90.00\end{array}$ \\
\hline Resolution $(\AA)$ & $\begin{array}{l}50.00-3.30 \\
(3.38-3.30)\end{array}$ & $\begin{array}{l}50.00-3.25 \\
(3.45-3.25)\end{array}$ & $\begin{array}{l}50.00-2.10 \\
(2.23-2.10)\end{array}$ & $\begin{array}{l}50.00-2.58 \\
(2.74-2.58)\end{array}$ & $\begin{array}{l}50.00-1.85 \\
(1.96-1.85)\end{array}$ & $\begin{array}{l}50.00-1.35 \\
(1.43-1.35)\end{array}$ & $\begin{array}{l}50.00-1.30 \\
(1.38-1.30)\end{array}$ & $\begin{array}{l}50.00-1.70 \\
(1.80-1.70)\end{array}$ & $\begin{array}{l}50.00-1.50 \\
(1.59-1.50)\end{array}$ & $\begin{array}{l}50.00-1.60 \\
(1.70-1.60)\end{array}$ & $\begin{array}{l}50.00-1.45 \\
(1.54-1.45)\end{array}$ & $\begin{array}{l}50.00-2.91 \\
(3.08-2.91)\end{array}$ \\
\hline$R_{\text {meas }}(\%)$ & $20.1(280.0)$ & $12.4(199.1)$ & $11.2(141.5)$ & $11.1(176.5)$ & $20.5(160.6)$ & $12.8(164.5)$ & $13.5(203.2)$ & $16.7(154.2)$ & $9.5(164.1)$ & $12.6(146.2)$ & $5.9(202.4)$ & $15.3(217.2)$ \\
\hline$<I / \sigma I>$ & $8.38(0.77)$ & $11.11(0.91)$ & $13.07(1.09)$ & $9.15(0.86)$ & $8.69(1.26)$ & $8.96(1.05)$ & $12.10(1.12)$ & $14.87(1.64)$ & $9.07(0.78)$ & $11.86(1.31)$ & $16.29(0.88)$ & $12.83(0.83)$ \\
\hline CC $1 / 2(\%)$ & $99.7(28.3)$ & $99.9(34.1)$ & $99.9(38.7)$ & $99.7(36.1)$ & $99.6(49.9)$ & $99.7(42.1)$ & $99.9(50.8)$ & $99.9(64.8)$ & $99.8(32.4)$ & $99.8(55.2)$ & $100(46.7)$ & $99.8(48.4)$ \\
\hline $\begin{array}{l}\text { Completeness } \\
(\%)\end{array}$ & $95.4(95.7)$ & $99.2(98.2)$ & $99.4(97.1)$ & $98.5(93.5)$ & $99.8(99.5)$ & $97.4(94.2)$ & $99.5(97.6)$ & $99.9(99.5)$ & $97.4(95.6)$ & $98.8(97.6)$ & $99.9(99.5)$ & $99.5(97.8)$ \\
\hline Redundancy & $7.5(7.5)$ & $4.5(4.5)$ & $6.1(5.1)$ & $3.3(3.2)$ & $9.2(9.2)$ & $6.9(6.8)$ & $10.3(10.3)$ & $15.3(15.4)$ & $3.5(3.5)$ & $6.8(6.9)$ & $6.7(6.0)$ & $6.5(6.4)$ \\
\hline Wilson B $\left(\AA^{2}\right)$ & 106.72 & 97.57 & 43.11 & 68.91 & 18.31 & 13.00 & 11.56 & 25.82 & 27.85 & 14.32 & 20.88 & 80.84 \\
\hline \multicolumn{13}{|l|}{ Refinement } \\
\hline Resolution $(\AA)$ & $49.00-3.30$ & $47.83-3.25$ & $39.00-2.10$ & $47.50-2.58$ & $61.94-1.85$ & $47.85-1.35$ & $48.72-1.30$ & $34.00-1.70$ & $48.53-1.50$ & $45.62-1.60$ & $47.94-1.45$ & $47.60-2.91$ \\
\hline No. reflections & 176285 & 99634 & 138531 & 144937 & 100130 & 458802 & 160728 & 71480 & 332071 & 270868 & 63952 & 48218 \\
\hline$R_{\text {work }} / R_{\text {free }}(\%)$ & $15.58 / 18.71$ & $16.16 / 19.25$ & $17.94 / 20.79$ & $18.33 / 21.63$ & $16.50 / 19.32$ & $15.62 / 17.43$ & $16.67 / 17.85$ & $17.28 / 19.21$ & $19.39 / 21.12$ & $15.67 / 17.73$ & $14.48 / 16.22$ & $18.93 / 22.29$ \\
\hline \multicolumn{13}{|l|}{ No. non-H atoms } \\
\hline Protein & 64032 & 31721 & 15704 & 27964 & 8104 & 16312 & 4106 & 3960 & 15373 & 15543 & 2006 & 11552 \\
\hline Ligand/ion & 760 & 320 & 177 & 497 & 107 & 478 & 122 & 71 & 114 & 288 & 58 & 149 \\
\hline Water & 16 & 0 & 724 & 254 & 593 & 1713 & 438 & 475 & 1284 & 1066 & 211 & 0 \\
\hline \multicolumn{13}{|l|}{$B$-factors $\left(\AA^{2}\right)$} \\
\hline Protein & 127.39 & 137.05 & 64.03 & 93.32 & 28.57 & 19.20 & 17.20 & 20.39 & 28.21 & 27.29 & 34.64 & 99.24 \\
\hline Ligand/ion & 126.12 & 138.21 & 52.79 & 107.16 & 50.12 & 26.54 & 20.44 & 40.05 & 30.50 & 28.83 & 74.68 & 96.35 \\
\hline Water & 98.04 & - & 56.89 & 70.70 & 36.60 & 30.49 & 27.22 & 32.98 & 34.11 & 32.52 & 47.12 & - \\
\hline \multicolumn{13}{|l|}{ R.m.s. deviations } \\
\hline Bond lengths $(\AA)$ & 0.010 & 0.010 & 0.010 & 0.010 & 0.010 & 0.010 & 0.010 & 0.010 & 0.010 & 0.010 & 0.010 & 0.010 \\
\hline Bond angles $\left({ }^{\circ}\right)$ & 1.16 & 1.13 & 1.07 & 1.17 & 1.01 & 1.11 & 1.05 & 0.92 & 0.95 & 1.01 & 1.03 & 1.21 \\
\hline Pdb id & $6 \mathrm{hxh}$ & $6 \mathrm{qfb}$ & $6 \mathrm{hxi}$ & $6 \mathrm{hxj}$ & $6 \mathrm{hxk}$ & $6 \mathrm{hxl}$ & $6 \mathrm{hxm}$ & $6 \mathrm{hxn}$ & $6 \mathrm{hxo}$ & $6 \mathrm{qcl}$ & 6 hxp & $6 \mathrm{hxq}$ \\
\hline
\end{tabular}

For each of the determined crystal structures, the crystallization condition, cryoprotectant, and data collection and refinement statistics are reported. Each dataset was collected from a single crystal. Values in parentheses are for the highest-resolution shell. 
Extended Data Table 2 | SAXS data collection and scattering-derived parameters

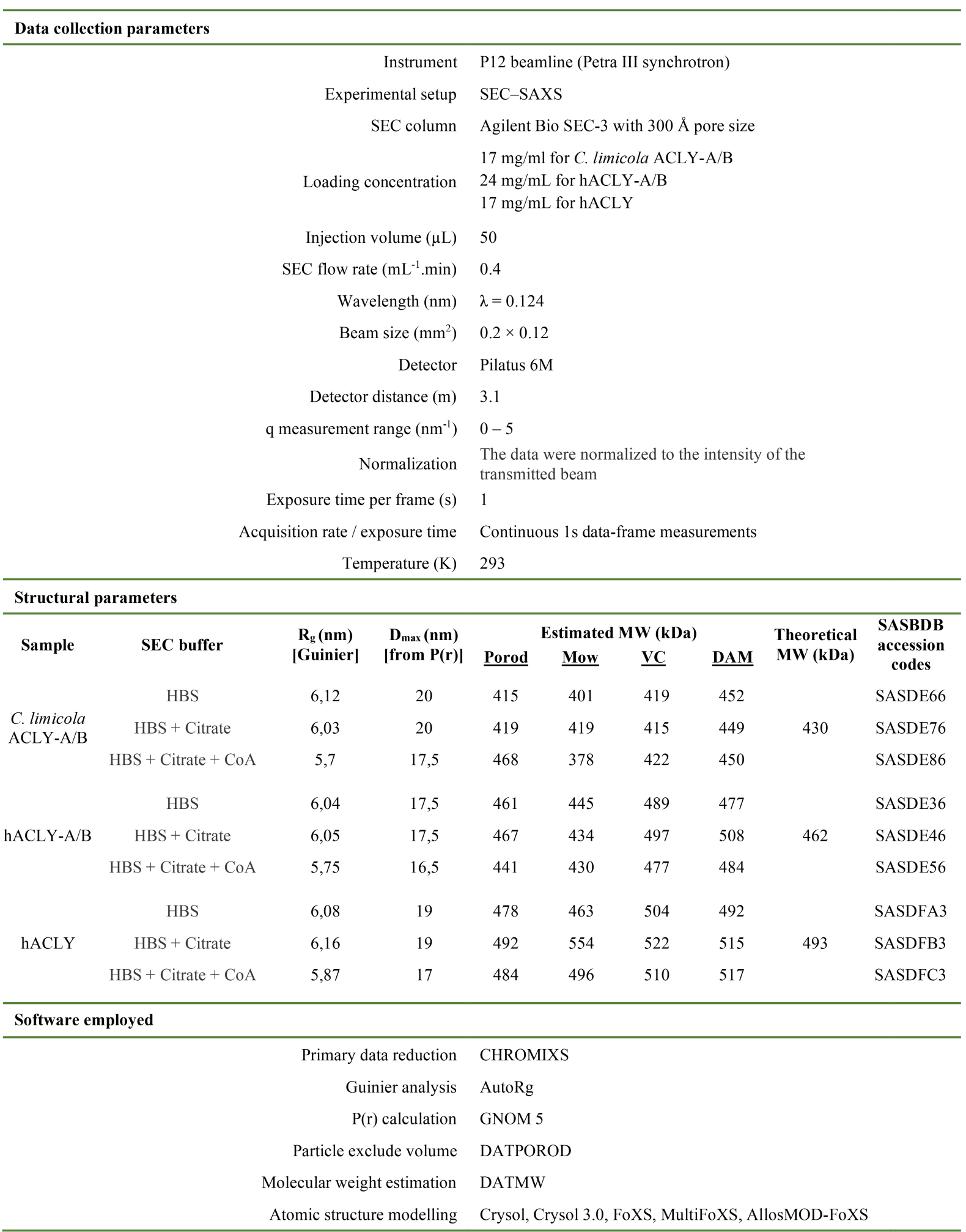




\section{Reporting Summary}

Nature Research wishes to improve the reproducibility of the work that we publish. This form provides structure for consistency and transparency in reporting. For further information on Nature Research policies, see Authors \& Referees and the Editorial Policy Checklist.

\section{Statistical parameters}

When statistical analyses are reported, confirm that the following items are present in the relevant location (e.g. figure legend, table legend, main text, or Methods section).

\begin{tabular}{l|l}
$\mathrm{n} / \mathrm{a}$ & Confirmed \\
$\square$ & $\bigotimes$ The exact sample size $(n)$ for each experimental group/condition, given as a discrete number and unit of measurement
\end{tabular}

$\square$ \ An indication of whether measurements were taken from distinct samples or whether the same sample was measured repeatedly

$\square \bigotimes \begin{aligned} & \text { The statistical test(s) used AND whether they are one- or two-sided } \\ & \text { Only common tests should be described solely by name; describe more complex techniques in the Methods section. }\end{aligned}$

Х $\square$ A description of all covariates tested

Х $\square$ A description of any assumptions or corrections, such as tests of normality and adjustment for multiple comparisons

$\triangle \square \begin{aligned} & \text { A full description of the statistics including central tendency (e.g. means) or other basic estimates (e.g. regression coefficient) AND } \\ & \text { variation (e.g. standard deviation) or associated estimates of uncertainty (e.g. confidence intervals) }\end{aligned}$

$\varnothing \square$ For null hypothesis testing, the test statistic (e.g. $F, t, r$ ) with confidence intervals, effect sizes, degrees of freedom and $P$ value noted

Give $P$ values as exact values whenever suitable.

Х $\square$ For Bayesian analysis, information on the choice of priors and Markov chain Monte Carlo settings

\ $\square$ For hierarchical and complex designs, identification of the appropriate level for tests and full reporting of outcomes

$\bigotimes \square$ Estimates of effect sizes (e.g. Cohen's $d$, Pearson's $r$ ), indicating how they were calculated

$\varnothing$ Clearly defined error bars

State explicitly what error bars represent (e.g. SD, SE, CI)

Our web collection on statistics for biologists may be useful.

\section{Software and code}

Policy information about availability of computer code $\begin{array}{ll}\text { Data collection } & \text { Crystallographic and SAXS data were collected at the following European synchrotron radiation facilities: Petra III (beamlines P12, P13 } \\ \text { and P14), SOLEIL (beamlines SWING and Proxima-2A), ESRF Grenoble (beamlines ID23-1, ID-23-2, ID30-B) and SLS (beamlines PXI and }\end{array}$ PXIII). Negative stain electron microscopy data were collected at the Electron Microscopy Platform of the IBS (Grenoble). Enzymatic assays and SEC-MALLS data were measured with a FLUOstar Omega microplate reader (BMG Labtech) and miniDAWN TREOS instrument (Wyatt), respectively, at the VIB-UGent Center for Inflammation Research (Zwijnaarde).

Data analysis

$$
\begin{aligned}
& \text { Software used to process, analyse and validate the crystallographic data was provided by the XDS-suite (multiple versions during } \\
& \text { 2016-2018), the CCP4 package version } 7.0 \text { (including AIMLESS, PHASER and PARROT), PHENIX version } 1.12 \text { (including phenix.refine and } \\
& \text { phenix.xtriage), autoBuster version 2.10.3 (Global Phasing Ltd.) and the PDB_REDO server. Structures were (re)build using Coot (version } \\
& \text { 0.8.8). SEC-MALS results were analysed using Astra 6.1 (Wyatt Technology). SAXS data was analyzed using programs from the ATSAS suite } \\
& \text { (version 2.8.4, including Chromixs, Crysol, Crysol 3.0, DATMW, AutoRg and GNOM 5), Foxtrot (version 3.3.4) and the FoXS, MultiFoXS and } \\
& \text { AllosMod-FoXS web servers. Single-particle negative stain electron microscopy analysis was performed using BOXER and RELION2.1. } \\
& \text { Kinetic data was analysed using GraphPad Prism 5. Structural superpositions were made with Chimera (version 1.10.1). Structures were } \\
& \text { analyzed with Pymol version 2.0. Secondary structure of protein models was assigned using the DSSP web server (version 3.0). Sequence } \\
& \text { alignments were created and edited using the ClustalOmega 1.2.4 and ESPript 3.0 web servers. }
\end{aligned}
$$


Policy information about availability of data

All manuscripts must include a data availability statement. This statement should provide the following information, where applicable:

- Accession codes, unique identifiers, or web links for publicly available datasets

- A list of figures that have associated raw data

- A description of any restrictions on data availability

Protein expression constructs generated in this study are available via the BCCM/GeneCorner Plasmid Collection (http://bccm.belspo.be) through the following accession codes: LMBP 11277 (pTrcHis2-hACL), LMBP 11131 (pET-DUET-hACL-A/B), LMBP 11132 (pET-Duet-Mco-ACL-A/B), LMBP 11133 (pET11a-Hth-CCL), LMBP 11134 (pET-Duet-Hth-CCS), LMBP 11125 (pET11a-Cli-ACL-A/B), LMBP 11128 (pET15b-hCCL) and LMBP 11129 (pET15b-Cli-CCL). X-ray crystallographic coordinates and structure factors have been deposited in the Protein Data Bank (PDB) with accession codes 6hxh (hACL-A/B in space group P1), 6qfb (hACL-A/B in space group C2), 6hxi (M. concilii ACL-A/B), 6hxj (C. limicola ACL-A/B), 6hxk (CCL module of hACL, space group P212121), 6hxl (CCL module of hACL, space group P21), 6hxm (CCL module of hACL, space group C2221), 6hxn (CCL module of C. limicola ACL, space group P3121), 6hxo (CCL module of C. limicola ACL, space group P21), 6qcl (CCL module of C. limicola ACL in complex with acetyl-CoA and L-malate), 6hxp (H. thermophilus CCL) and 6hxq (H. thermophilus CCS). SAXS data and models have been deposited in the Small Angle Scattering Biological Data Bank with accession codes SASDE36, SASDE46 and SASDE56 for hACL-A/B; SASDFA3, SASDFB3 and SASDFC3 for hACL; and SASDE66, SASDE76 and SASDE86 for C. limocola ACL-A/B. Source data for the enzymatic assays for hACL and hACL-A/B (Extended Data Fig. 1d) and the SEC-MALLS analysis for hACL-A/B (Extended Data Fig. 1e) are available via the online version of this manuscript.

\section{Field-specific reporting}

Please select the best fit for your research. If you are not sure, read the appropriate sections before making your selection.

$\bigotimes$ Life sciences $\quad \square$ Behavioural \& social sciences $\quad \square$ Ecological, evolutionary \& environmental sciences

For a reference copy of the document with all sections, see nature.com/authors/policies/ReportingSummary-flat.pdf

\section{Life sciences study design}

All studies must disclose on these points even when the disclosure is negative.

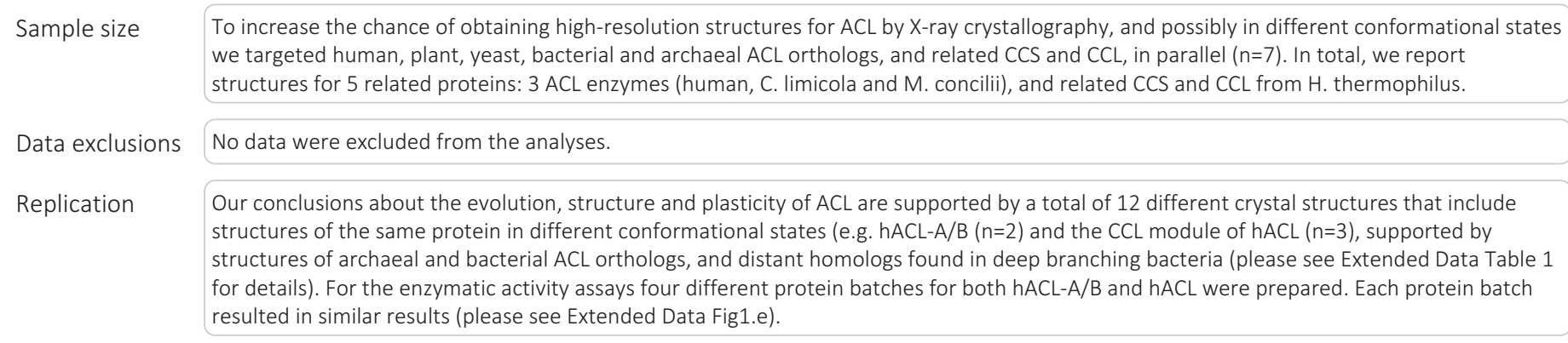

Randomization Our study did not involve experiments were randomization of samples was required or appropriate.

Blinding Our study did not involve experiments were blinding was required or appropriate.

\section{Reporting for specific materials, systems and methods}

Materials \& experimental systems

\begin{tabular}{l|l}
\hline & Involved in the study \\
$\square$ & $\square$ Unique biological materials \\
$\square$ & Antibodies \\
$\square$ & Eukaryotic cell lines \\
$\square$ Human research participants
\end{tabular}

\begin{tabular}{l|l}
\multicolumn{2}{l}{ Methods } \\
\hline n/a & Involved in the study \\
$\square$ & $\square$ ChIP-seq \\
\hline & $\square$ Flow cytometry
\end{tabular}

